\title{
Instantaneous Gratification
}

\section{Citation}

Harris, Christopher and Dan Laibson. Forthcoming. Instantaneous gratification. Quarterly Journal of Economics.

\section{Permanent link}

http://nrs.harvard.edu/urn-3:HUL.InstRepos:9918802

\section{Terms of Use}

This article was downloaded from Harvard University's DASH repository, and is made available under the terms and conditions applicable to Open Access Policy Articles, as set forth at http:// nrs.harvard.edu/urn-3:HUL.InstRepos:dash.current.terms-of-use\#OAP

\section{Share Your Story}

The Harvard community has made this article openly available.

Please share how this access benefits you. Submit a story.

Accessibility 


\title{
INSTANTANEOUS GRATIFICATION*
}

\author{
By Christopher Harris and David Laibson
}

This Draft: June 14, 2012

\begin{abstract}
Extending Barro (1999) and Luttmer \& Mariotti (2003), we introduce a new model of time preferences: the instantaneous-gratification model. This model applies tractably to a much wider range of settings than existing models. It applies to both complete- and incomplete-market settings and it works with generic utility functions. It works in settings with linear policy rules and in settings in which equilibrium cannot be supported by linear rules. The instantaneous-gratification model also generates a unique equilibrium, even in infinite-horizon applications, thereby resolving the multiplicity problem hitherto associated with dynamically inconsistent models. Finally, it simultaneously features a single welfare criterion and a behavioral tendency towards overconsumption.
\end{abstract}

Word count: 9948

JEL codes: C6, C73, D91, E21.

Keywords: quasi-hyperbolic discounting, time preference, dynamic inconsistency, continuous time, consumption, savings, buffer stock, Euler Relation, dynamic games, altruistic growth.

*We thank Robert Barro, Drew Fudenberg, Elhanan Helpman, George Mailath, Andrew Postlewaite, three anonymous referees, and seminar participants at European Summer Symposium on Economic Theory (Gerzensee), Harvard University, Federal Reserve Bank of Minneapolis, Princeton University, Stanford University, Universita Bocconi, University of California Berkeley, University of Chicago, University College London, University of Minnesota, University of Pennsylvania, Universitat Pompeu Fabra, Wharton, and Yale University. Alfred Galichon, Evdokia Nikolova, Nicholas Roussanov, Laura Serban, and Jann Spiess provided outstanding research assistance. Harris acknowledges financial support from the British Academy. Laibson acknowledges financial support from the National Science Foundation (SBR-9510985), the Olin Foundation, the National Institute on Aging (R01-AG-1665 and P01-AG-005842 ) and the MacArthur Foundation. Corresponding author is Christopher Harris: Faculty of Economics, University of Cambridge, Austin Robinson Building, Sidgwick Avenue, Cambridge, CB3 9DD, United Kingdom. Email: cjharris@econ.cam.ac.uk. 


\section{IntroduCtion}

The discrete-time quasi-hyperbolic discount function $\left\{1, \beta \delta, \beta \delta^{2}, \beta \delta^{3}, \ldots\right\}$ is used to model high rates of short-run discounting. ${ }^{1}$ With $\beta<1$, this present-biased discount function generates a gap between a high short-run discount rate $(-\ln \beta \delta)$ and a low long-run rate $(-\ln \delta)$. The quasi-hyperbolic discount function has been used to study a range of behaviors, including consumption, procrastination, addiction and search. ${ }^{2}$

Extending the work of Barro (1999) and Luttmer and Mariotti (2003) on continuoustime models of non-exponential discounting, the current paper shows how to operationalize quasi-hyperbolic time preferences in continuous time. Our model - which we call the instantaneous-gratification model or IG model - applies tractably to a much wider range of settings than existing models. It applies to incomplete-market settings in which liquidity constraints arise because future labor income can't be used as collateral; and it works with an economically rich class of utility functions which is much larger than the class with constant relative risk aversion. Consequently, we do not need to restrict analysis to linear policy rules or to settings in which such rules support an equilibrium.

We develop the IG model in two steps. In the first step, following Barro and LuttmerMariotti, we assume that the present is valued discretely more than the future, mirroring the one-time drop in valuation implied by the discrete-time quasi-hyperbolic discount function. However, we assume that the transition from the present to the future occurs with a constant hazard rate $\lambda$. This assumption reduces the Bellman equation to a pair of stationary differential equations that characterize the current- and continuation-value functions. We call the resulting model the present-future model or PF model.

In the second step, we let the hazard rate $\lambda$ of transitions from present to future go to $\infty$. This brings us to the IG model. The Bellman equation for the IG model is even simpler than that of the PF model: it is a single ordinary differential equation.

Using convex duality we characterize the solution of the IG model. We prove and then exploit the fact that the value function of the IG model is identical to the value function of an optimization problem with (i) the same long-run discount rate as the IG model, and (ii) a different flow utility function that depends on both the level of consumption and the level of financial assets.

\footnotetext{
${ }^{1}$ See Phelps and Pollak (1968) and Laibson (1997). Strotz (1956) first formalized the idea that the short-run discount rate is greater than the long-run discount rate.

${ }^{2}$ For example, see Akerlof (1991), O’Donoghue and Rabin (1999), Angeletos, Laibson, Repetto, Tobacman and Weinberg (2001), DellaVigna and Malmendier (2004), and Della Vigna and Paserman (2005).
} 
Hence the IG model, which is dynamically inconsistent, has the same value function as a non-standard but dynamically consistent optimization problem. The IG model is not, however, observationally equivalent to this optimization problem: the IG model and the optimization problem share the same long-run discount rate and value function, but they have different instantaneous utility functions and equilibrium policies. ${ }^{3}$ The non-standard optimization problem is interesting, not because we think it is psychologically relevant, but rather because its partial equivalence enables us to use the machinery of optimization to study the value function of a dynamically inconsistent problem.

The IG model therefore carves out a tractable niche between dynamically inconsistent models and dynamically consistent models. On the one hand, it features dynamically inconsistent behavior and rational expectations. So, at each moment, the individual acts strategically with regard to her future preferences. On the other hand, the fact that the IG value function coincides with the value function of a related optimization problem implies that the IG model inherits many standard regularity properties.

For example, the value-function-equivalence result implies that the IG model has a unique equilibrium. This uniqueness result is surprising, since the quasi-hyperbolic model is a dynamic game. Indeed, Krusell and Smith (2000) have shown that Markov-perfect equilibria are not unique in a deterministic discrete-time setting. In contrast, we provide two uniqueness results. First, we prove uniqueness in the case in which asset returns are stochastic. Second, we show that the unique equilibrium of the stochastic IG model converges to an equilibrium of the corresponding deterministic model as the noise in the asset returns goes to zero. In other words, we are able to select a unique equilibrium of the deterministic IG model by using a standard equilibrium-refinement procedure. ${ }^{4}$

Similarly, we can give a detailed characterization of the consumption function in the IG model. When the expected rate of return is below a key threshold, the equilibrium consumption function displays a discontinuity at the liquidity constraint. Consequently, consumption falls discontinuously when a consumer spends down her assets and hits the liquidity constraint. This intuitive prediction is not possible in dynamically-consistent consumption models, which imply that the timepath of consumption is continuous, even at the point at which the consumer hits a liquidity constraint.

\footnotetext{
${ }^{3}$ See Laibson (1997) and Barro (1999) for cases with observational equivalence of policy functions.

${ }^{4}$ Our uniqueness result even offers something new in settings in which linear policy rules support an equilibrium: it tells us that if one can find an equilibrium in linear policy rules then that equilibrium is unique in the set of all policy rules, linear or non-linear.
} 
Finally, the IG model features a single welfare criterion, even though the model involves dynamically inconsistent behavioral choices. Because the present is valued discretely more than the future, the current self has an incentive to overconsume; but the discretely higher value of the present only lasts for an instant, so this overvaluation does not affect the welfare criterion. Hence, the model simultaneously features a single welfare function and a behavioral tendency toward overconsumption.

In summary, the IG model is generalizable with regard to both market completeness and consumption preferences, supports a unique equilibrium, makes new predictions about the consumption function, and identifies a single sensible welfare criterion.

In Section 2 we present the PF model of time preferences. In Section 3 we present the consumption problem that we use as our application. In Section 4 we describe the IG model, which arises when we start with the PF model and let the hazard rate of transition from present to future go to infinity. In Section 5 we show that the IG model has the same value function as a related, but non-standard, dynamically-consistent optimization problem. We use this partial equivalence result to prove equilibrium existence and uniqueness. We also use it to derive a unique equilibrium of the limiting version of our model in which the noise in the economy goes to zero. In Section 6, we characterize the equilibrium consumption function for the limiting case of no labor income. In Section 7 , we characterize the equilibrium consumption function for the general case with labor income. Section 8 concludes.

\section{The Present-Future Model of Time Preferences}

We now describe a class of discount functions that model present-biased preferences in continuous time. There are two alternative representations: a stochastic discount function, which we present first, and a deterministic discount function.

2.1. A Stochastic Discount Function. In the discrete-time formulation of quasihyperbolic time preferences, it is natural to divide time into two intervals: the present consisting of only the current period - and the future. All periods, present and future, are discounted exponentially with the discount factor $0<\delta<1$. Future periods are further discounted with uniform weight $0<\beta \leq 1$. Combining these pieces, the present period (i.e. $t=0$ ) receives full weight, and future periods (i.e. $t \geq 1$ ) are given weight $\beta \delta^{t}$.

This model can be generalized in two ways. First, the present could last for an arbitrary length of time, instead of ending after the current period. Second, the duration of the 
present could be stochastic, instead of being deterministic. Both of these generalizations have natural continuous-time analogues.

Consider an economic self born at time $s_{0}=0$. Call this self 'self 0 '. The lifetime of self 0 is divided into two intervals: a 'present', which lasts from $s_{0}$ to $s_{0}+\tau_{0}$; and a 'future', which lasts from $s_{0}+\tau_{0}$ to $\infty$. Think of the present as the interval during which control is exercised by self 0 , and of the future as the interval during which control is exercised by subsequent selves. The length $\tau_{0}$ of the present is stochastic, and is distributed exponentially with hazard rate $\lambda \in[0, \infty)$.

When the present of self 0 ends at $s_{0}+\tau_{0}$, a new self is born and takes control of decision-making. Call this new arrival 'self 1'. The preferences of self 1 , like those of self 0 , can be divided into two intervals. Self 1 has a present that lasts from $s_{1}=s_{0}+\tau_{0}$ to $s_{1}+\tau_{1}$, and a future that lasts from $s_{1}+\tau_{1}$ to $\infty$. Continuing in this way, we obtain a sequence of selves $\{0,1,2, \ldots\}$ born at dates $\left\{s_{0}, s_{1}, s_{2}, \ldots\right\}$. For all $n \geq 1$, self $n$ has a present that lasts from $s_{n}=s_{n-1}+\tau_{n-1}$ to $s_{n}+\tau_{n}$, and a future that lasts from $s_{n}+\tau_{n}$ to $\infty$. Figure 1 provides a visual representation.

We assume that all selves discount exponentially with discount factor $0<\delta<1$. Furthermore, each self values her future discretely less than her present, discounting it by the additional factor $0<\beta \leq 1$. More explicitly, we assume that self $n$ applies the discount factor $D_{n}(t)$ to the utility flow at time $s_{n}+t$, where

$$
D_{n}(t)=\left\{\begin{array}{lll}
\delta^{t} & \text { if } & t \in\left[0, \tau_{n}\right) \\
\beta \delta^{t} & \text { if } & t \in\left[\tau_{n}, \infty\right)
\end{array}\right\} .
$$

In other words, her discount function $D_{n}$ decays exponentially at rate $\gamma=-\ln \delta$ up to time $\tau_{n}$, drops discontinuously at $\tau_{n}$ to a fraction $\beta$ of its level just prior to $\tau_{n}$, and decays exponentially at rate $\gamma$ thereafter. ${ }^{5}$ Figure 2 plots a single realization of this discount function, with $\tau_{n}=3.4$.

This continuous-time discount function nests exponential discounting: either set $\lambda=0$, so that the future never arrives; or set $\beta=1$, so that there is no distinction between present and future. It is similar to some of the deterministic discount functions used in Barro (1999) and Luttmer and Mariotti (2003). However, we assume that $\tau_{n}$ is stochastic. Among other things, this ensures that the expectation of the discount function is smooth.

\footnotetext{
${ }^{5}$ The lengths $\left\{\tau_{0}, \tau_{1}, \tau_{2}, \ldots\right\}$ of the present intervals are i.i.d.
} 
When $\lambda \rightarrow \infty$, the discount function $D_{n}$ converges to the limiting function:

$$
D_{\infty}(t)=\left\{\begin{array}{ll}
1 & \text { if } t=0 \\
\beta \delta^{t} & \text { if } t \in(0, \infty)
\end{array}\right\}
$$

Characterizing this limiting case is the main focus of the current paper. ${ }^{6}$

2.2. A Reinterpretation Using a Deterministic Discount Function. The arguments in this paper are consistent with a second interpretation of the time preferences described above: one can assume that a new self is born every instant; that the present of each self lasts only an instant; and that each self has a deterministic discount function $\bar{D}$ equal to the expected value of the stochastic discount function $D_{n}$ described above (Harris and Laibson 2001). ${ }^{7}$ We describe this alternative deterministic interpretation in the current subsection and compare it with the stochastic approach in Subsection 2.3. Readers who wish to skip this material, can jump immediately to Section 3 without loss of continuity.

In the deterministic interpretation, each self uses the discount function $\bar{D}$ given by

$$
\bar{D}(t)=\mathrm{E}\left[D_{n}(t)\right]=e^{-\lambda t} \delta^{t}+\left(1-e^{-\lambda t}\right) \beta \delta^{t} .
$$

$\bar{D}(t)$ is the sum of two terms. The first term is the probability $e^{-\lambda t}$ that the drop in $D_{n}$ does not occur before time $t$, times the discount factor $\delta^{t}$ that applies before the drop. The second term is the probability $1-e^{-\lambda t}$ with which the drop in $D_{n}$ occurs after time $t$, times the discount factor $\beta \delta^{t}$ that applies after the drop. $\bar{D}(t)$ can also be written

$$
\bar{D}(t)=(1-\beta) e^{-(\gamma+\lambda) t}+\beta e^{-\gamma t}
$$

where $\gamma=-\ln (\delta)>0$ is the long-run discount rate. Written this way, $\bar{D}(t)$ is seen to be a convex combination of the short-run exponential discount factor $e^{-(\gamma+\lambda) t}$, with weight $1-\beta$, and the long-run exponential discount factor $e^{-\gamma t}$, with weight $\beta$.

The instantaneous discount rate of the deterministic discount function $\bar{D}$ is

$$
-\frac{\bar{D}^{\prime}(t)}{\bar{D}(t)}=\gamma+\frac{\lambda e^{-\lambda t}(1-\beta) \delta^{t}}{\bar{D}(t)} .
$$

\footnotetext{
${ }^{6}$ Notice that, because $\tau_{n} \rightarrow 0$ as $\lambda \rightarrow \infty$, the expectation of the discount function - which is smooth when $\lambda$ is finite - has a discontinuity when $\lambda=\infty$. This does not cause any problems.

${ }^{7}$ See footnote 15 for a development of this line of argument.
} 
It too is a sum of two terms. The first term is the long-run (exponential) discount rate $\gamma$. The second term is the expected drop in $D$ at time $t$, namely $\lambda e^{-\lambda t}(1-\beta) \delta^{t}$, divided by expected value of $D$ at time $t$, namely $\bar{D}(t)$. (The flow probability of a drop at time $t$ is $\lambda e^{-\lambda t}$ and the size of the drop is $(1-\beta) \delta^{t}$.) Note that the instantaneous discount rate falls from $\gamma+\lambda(1-\beta)$ at $t=0$ to $\gamma$ at $t=\infty$. Figure 3 plots $\bar{D}$ for $\lambda \in\{0,0.1,1, \infty\}$.

\subsection{Comparison of the Stochastic and Deterministic Discount Functions.}

The stochastic and deterministic discount functions differ in one key way: the stochastic discount function assumes a present of non-infinitesimal duration $\tau_{n}>0$, whereas the deterministic discount function assumes a present of infinitesimal duration $d t$. Hence the stochastic discount function assumes a countable number of non-infinitesimal selves, while the deterministic discount function assumes a continuum of infinitesimal selves.

The two formulations generate the same equilibrium behavior. To see why, note that the current self in the stochastic formulation is dynamically consistent during her period of control between time $s_{n}$ and time $s_{n+1}=s_{n}+\tau_{n}$. It therefore makes no difference whether we regard her as a non-infinitesimal agent, who decides how to behave at the outset of her control interval, or as a continuum of infinitesimal agents, each of which makes a decision during its instant of control.

The stochastic formulation has two advantages over the deterministic one. First, it can be set up using only standard mathematical tools. Second, when the stochastic formulation is used, we can derive the IG model in a single step. ${ }^{8}$ We therefore focus on the stochastic formulation.

\section{Applichtion to a Consumption Problem}

We now describe an important economic environment that we use to illustrate the implications of the discounting model. We study an infinite-horizon consumption-savings problem with liquidity constraints (cf. Deaton 1991, Carroll 1992). We include liquidity constraints, since they make a fundamental difference to the analysis by necessitating non-linear policy rules. On the other hand, we exclude labor-income uncertainty, since that would complicate the notation and does not affect our conclusions. This section also defines equilibrium and introduces the Bellman-system representation.

\footnotetext{
${ }^{8}$ In the analysis using the stochastic discount function, we let $\lambda \rightarrow \infty$. In doing so, we simultaneously pass from non-infinitesimal to infinitesimal selves and from the finite- $\lambda$ discount function to the infinite- $\lambda$ discount function. By contrast, in order to set up the deterministic discount function, we first have to formalize the idea of an infinitesimal self. This involves taking the limit as the span of control of a non-infinitesimal self goes to 0 . We then let $\lambda \rightarrow \infty$.
} 
3.1. The Dynamics. At any time $t \geq 0$, the consumer has a stock of financial wealth $x \geq 0$ and receives a flow of labor income $y>0$. If $x>0$, she can choose any consumption level $c>0$ : wealth is a stock and consumption is a flow, so any finite consumption is achievable provided it is not maintained for too long. If $x=0$ then she can only choose a consumption level $0<c \leq y$ : she has no wealth and cannot borrow, so she cannot consume more than her labor income. This is the liquidity constraint.

Whatever the consumer does not consume is invested in an asset, the returns on which are distributed normally with mean $\mu d t$ and variance $\sigma^{2} d t$, where $\mu \in(-\infty, \infty)$ and $\sigma \in(0, \infty)$. The change in wealth is therefore

$$
d x=(\mu x+y-c) d t+\sigma x d z
$$

where $z$ is a standard Wiener process. ${ }^{9}$

3.2. Equilibrium. Recall that the consumer is modeled as a sequence of autonomous selves (see Figure 1). Each self controls consumption during her own present and cares about - but does not control - consumption in her future. Our consumption problem is therefore an intrapersonal game. Following the literature in intergenerational games, our solution concept for this game will be stationary Markov-perfect equilibrium. ${ }^{10}$

Maskin and Tirole (2001) define Markov-perfect equilibrium (or MPE for short). MPE is a refinement of subgame-perfect equilibrium which only allows strategies to depend on information that is directly payoff relevant (i.e. information that is necessary to determine players' choice sets or payoffs). It does not allow strategies to depend on information that is only indirectly relevant (e.g. it does not allow the strategy of one player to depend on information that only becomes relevant if the strategy of another player depends on it). In our model, the only information that is directly payoff relevant is the current level of wealth, so MPE restricts analysis to strategies that map current wealth to consumption. ${ }^{11}$ We go further, restricting attention to stationary MPE (or SMPE for short). In other

\footnotetext{
${ }^{9}$ We could also make labor income stochastic: in addition to labor income flow $y$, the agent receives sporadic (i.i.d.) lump-sum bonuses. To preserve stationarity, such bonuses would arrive with a constant hazard. We could even allow for non-stationary labor income, at the expense of an extra state variable. These generalizations would not change our results.

${ }^{10}$ For two important examples, see Bernheim and Ray (1987) and Leininger (1986).

${ }^{11}$ In our model, the information available to self $n$ at time $t \in\left[s_{n}, s_{n+1}\right)$ consists of the timepath $z:[0, t] \rightarrow \mathbb{R}$ of past shocks, the timepath $x:[0, t] \rightarrow[0, \infty)$ of past wealth, the sequence $\left\{s_{0}, s_{1}, \ldots, s_{n}\right\}$ of past transition times, the timepath $c:[0, t) \rightarrow(0, \infty)$ of past consumption and the current time. Of all this information, only the current value of wealth $x_{t}$ is directly payoff relevant.
} 
words, we study equilibria in which all selves use the same strategy.

Consider self $n$. Suppose that the future selves $n+1, n+2, \ldots$ all employ the same Markov strategy $\widetilde{c}:[0, \infty) \rightarrow(0, \infty)$. Then the dynamics of wealth from time $s_{n}+\tau_{n}$ onwards are given by

$$
d x_{t}=\left(\mu x_{t}+y-\widetilde{c}\left(x_{t}\right)\right) d t+\sigma x_{t} d z
$$

and the continuation value of self $n$ is

$$
v\left(x_{s_{n}+\tau_{n}}, \widetilde{c}\right)=\mathrm{E}_{s_{n}+\tau_{n}}\left[\int_{s_{n}+\tau_{n}}^{\infty} e^{-\gamma\left(t-\left(s_{n}+\tau_{n}\right)\right)} u\left(\widetilde{c}\left(x_{t}\right)\right) d t\right],
$$

where: $s_{n}+\tau_{n}$ is the time at which control passes from self $n$ to self $n+1 ; x_{s_{n}+\tau_{n}}$ is wealth at time $s_{n}+\tau_{n} ; u:(0, \infty) \rightarrow \mathbb{R}$ is the instantaneous utility function; $\gamma=-\ln (\delta)>0$ is the long-run discount rate; and $\mathrm{E}_{s_{n}+\tau_{n}}$ denotes expectations conditional on the information available at time $s_{n}+\tau_{n} \cdot{ }^{12}$

Suppose further that self $n$ employs the Markov strategy $c:[0, \infty) \rightarrow(0, \infty)$. Then the dynamics of wealth from time $s_{n}$ to time $s_{n}+\tau_{n}$ are given by

$$
d x_{t}=\left(\mu x_{t}+y-c\left(x_{t}\right)\right) d t+\sigma x_{t} d z
$$

and the current value of self $n$ is

$$
w\left(x_{s_{n}}, c, \widetilde{c}\right)=\mathrm{E}_{s_{n}}\left[\int_{s_{n}}^{s_{n}+\tau_{n}} e^{-\gamma\left(t-s_{n}\right)} u\left(c\left(x_{t}\right)\right) d t+\beta e^{-\gamma \tau_{n}} v\left(x_{s_{n}+\tau_{n}}, \widetilde{c}\right)\right]
$$

where: $s_{n}$ is the time at which control passes from self $n-1$ to self $n ; x_{s_{n}}$ is wealth at time $s_{n}$; and $\mathrm{E}_{s_{n}}$ denotes expectations conditioned on information available at time $s_{n}$.

The objective of self $n$ is to find a Markov strategy $c^{*}$ that is optimal in the sense that, for all $x_{s_{n}} \geq 0, c^{*}$ maximizes $w\left(x_{s_{n}}, c, \widetilde{c}\right)$ with respect to $c{ }^{13}$ We denote by $\operatorname{BR}(\widetilde{c})$ the set of all such Markov strategies $c^{*}$. An SMPE of our model is then any Markov strategy $c$ such that $c \in \operatorname{BR}(c)$.

3.3. Bellman System for $v, w$ and $c$. An SMPE can be characterized in terms of three functions: the equilibrium Markov strategy (or consumption function) $c$ itself; the associated continuation-value function $v:[0, \infty) \rightarrow \mathbb{R}$; and the associated current-value

\footnotetext{
${ }^{12}$ Karatzas and Shreve (1991) discuss regularity conditions for stochastic differential equations.

${ }^{13}$ Given that all future selves are employing Markov strategies, a non-Markov strategy never does better for the current self than an optimal Markov strategy.
} 
function $w:[0, \infty) \rightarrow \mathbb{R}$.

Since future selves use the consumption function $c$, the continuation-value function $v$ must satisfy the differential equation

$$
0=\frac{1}{2} \sigma^{2} x^{2} v^{\prime \prime}+(\mu x+y-c) v^{\prime}-\gamma v+u(c)
$$

for $x \in[0, \infty)$, where we have suppressed the dependence of $v$ and $c$ on $x$. This equation reflects the fact that the following effects must sum to zero: the expected instantaneous change in the value function $\left(\frac{1}{2} \sigma^{2} x^{2} v^{\prime \prime}+(\mu x+y-c) v^{\prime}\right)$; the instantaneous change in value due to discounting $(-\gamma v)$; and the instantaneous utility flow $(u(c))$.

Similarly, since the current self also uses the consumption function $c$, and since the continuation-value function is $v$, the current-value function $w$ satisfies

$$
0=\frac{1}{2} \sigma^{2} x^{2} w^{\prime \prime}+(\mu x+y-c) w^{\prime}+\lambda(\beta v-w)-\gamma w+u(c)
$$

for $x \in[0, \infty)$, where we have suppressed the dependence of $v, w$ and $c$ on $x$. This equation is very similar to equation (2). The only differences are: (i) the current-value function $w$ replaces the continuation-value function $v$; and (ii) there is an additional term $\lambda(\beta v-w)$, which reflects the hazard rate $\lambda$ of making the transition from the present, valued by $w$, to the future, valued by $\beta$ times $v$.

Third, if self $n$ behaves optimally - taking the behavior of her future selves as given then $c$ will satisfy the instantaneous optimality condition

$$
\left\{\begin{array}{ll}
u^{\prime}(c)=w^{\prime} & \text { if } x>0 \\
u^{\prime}(c)=\max \left\{u^{\prime}(y), w^{\prime}\right\} & \text { if } x=0
\end{array}\right\} .
$$

Intuitively, if $x>0$, then there is no constraint on consumption. So consumption $c$ is chosen to equate the marginal utility of consumption $u^{\prime}(c)$ and the marginal value of current wealth $w^{\prime}$. If $x=0$, then the liquidity constraint may or may not bind: if $w^{\prime}<u^{\prime}(y)$, then the constraint binds, and $c=y$ (or, equivalently, $u^{\prime}(c)=u^{\prime}(y)$ ); if $w^{\prime} \geq u^{\prime}(y)$ then the constraint does not bind, and $u^{\prime}(c)=w^{\prime}$.

Fourth, systems of second-order ordinary differential equations like (2-4) typically require two boundary conditions. We have already supplied one boundary condition, by requiring that equations (2-4) hold at $x=0$, and not just in the interior of the wealth space. We refer to this as the boundary condition at 0 . But we need to supply a second 
boundary condition. This boundary condition has two parts: global upper bounds for $v$ and $w$, and global lower bounds for $v$ and $w$. Among other things, these bounds have the effect of controlling the behavior of $v$ and $w$ near infinity.

Let us assume temporarily that $u$ is bounded below. Then it is easy to see that $v$ is bounded below by $\frac{1}{\gamma} u(0)$, and that $w$ is bounded below by $\frac{\gamma+\beta \lambda}{\gamma(\gamma+\lambda)} u(0)$. Furthermore $v$ is bounded above by the value function $\bar{v}$ of a consumer who: (i) has utility function $u$; and (ii) discounts the future exponentially at rate $\gamma$. Finally, $w$ is bounded above by the value function $\bar{w}$ of a consumer who: (i) has utility function $\widetilde{u}=u+\max \{0,-u(0)\}$; and (ii) discounts the future exponentially at rate $\gamma \cdot{ }^{14}$

Putting these observations together, we have the following characterization of equilibrium in the $\mathrm{PF}$ model.

Theorem 1. Suppose that $u$ is bounded below. Then the consumption function $c:[0, \infty) \rightarrow(0, \infty)$ is an SMPE of the PF model if and only if there is a continuationvalue function $v:[0, \infty) \rightarrow \mathbb{R}$ and a current-value function $w:[0, \infty) \rightarrow \mathbb{R}$ such that $(c, v, w)$ together satisfy the pair of differential equations

$$
\begin{aligned}
& 0=\frac{1}{2} \sigma^{2} x^{2} v^{\prime \prime}+(\mu x+y-c) v^{\prime}-\gamma v+u(c), \\
& 0=\frac{1}{2} \sigma^{2} x^{2} w^{\prime \prime}+(\mu x+y-c) w^{\prime}+\lambda(\beta v-w)-\gamma w+u(c)
\end{aligned}
$$

for all $x \in[0, \infty)$, the optimality condition

$$
\left\{\begin{array}{ll}
u^{\prime}(c)=w^{\prime} & \text { if } x>0 \\
u^{\prime}(c)=\max \left\{u^{\prime}(y), w^{\prime}\right\} & \text { if } x=0
\end{array}\right\}
$$

and the global bounds

$$
\begin{aligned}
\frac{1}{\gamma} u(0) & \leq v \leq \bar{v} \\
\frac{\gamma+\beta \lambda}{\gamma(\gamma+\lambda)} u(0) & \leq w \leq \bar{w}
\end{aligned}
$$

for all $x \in[0, \infty)$. We refer to (5-9) as the Bellman system of the PF consumer. ${ }^{15}$

In general, the PF model can be expected to have a finite number of equilibria. Furthermore, if $\lambda$ is close to 0 (a dynamically consistent limit case), then equilibrium is

\footnotetext{
${ }^{14}$ This is because: (i) considering utility flows in the present, we have $u \leq \widetilde{u}$; and (ii) considering utility flows in the future, we have $\beta u \leq \beta \widetilde{u} \leq \widetilde{u}$.

${ }^{15}$ In the model with the deterministic discount function $\bar{D}$, the consumption function $c$ is a SMPE iff
} 
unique. Similarly, if $\beta$ is close to 1 (another dynamically consistent limit case), then equilibrium is again unique. Much more interestingly, if $\lambda$ is close to $\infty$ (a dynamically inconsistent limit case), then equilibrium is unique. This is the case that we study next.

\section{The Instantaneous-Gratification Model}

Experimental evidence suggests that the present - in other words, the interval $\left[s_{n}, s_{n}+\tau_{n}\right)$ during which consumption is not down-weighted by $\beta$ - is short. ${ }^{16}$ This is the same as saying that $\lambda$ is large, since the arrival rate of the future is $\lambda$. In the current section, we consider the limiting case $\lambda \rightarrow \infty$, which serves as an approximation of situations in which the duration of the present (namely $\tau$ ) is short. We refer to the limiting case as the instantaneous-gratification model, or IG model.

In Section 6 we show that $\lambda \rightarrow \infty$ is a good approximation for $\lambda \geq 12$. In other words, if time is measured in years and the average duration of the present is less than a month, then the IG model $(\lambda \rightarrow \infty)$ is a good approximation for the PF model (with $\lambda \geq 12$ ).

There are two ways to derive the IG model. The first way is to derive the Bellman equation of the IG consumer by taking the limit of the Bellman system of the PF consumer as $\lambda \rightarrow \infty$. This explicitly links the PF model and the IG model. Moreover, defining an equilibrium concept as the set of limit points of equilibria in a neighborhood of games is

there is a value function $V$ such that $(c, V)$ jointly satisfy the partial differential equation

$$
0=\frac{1}{2} \sigma^{2} x^{2} \frac{\partial^{2} V}{\partial x^{2}}(t, x)+(\mu x+y-c(x)) \frac{\partial V}{\partial x}(t, x)+\bar{D}(t) u(c(x))+\frac{\partial V}{\partial t}(t, x),
$$

the optimality conditions

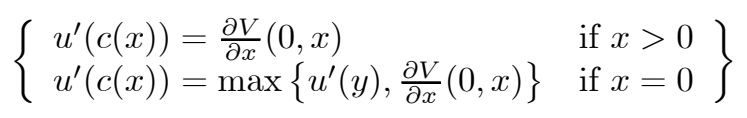

and the global bounds

$$
\left(\int_{t}^{\infty} \bar{D}(s) d s\right) u(0) \leq V(t, x) \leq \bar{V}(t, x) .
$$

for all $(t, x) \in[0, \infty)^{2}$. Here: $V(t, x)$ is the value at time 0 of consumption over the interval $[t, \infty)$ when financial wealth is $x ; \frac{\partial V}{\partial x}(0, x)$ is the marginal value of wealth at time 0 when financial wealth is $x$; and $\bar{V}$ is the value function of a time-consistent consumer with discount function $\bar{D}$. We refer to this as the Bellman system of the $\bar{D}$ consumer. It is valid for general $\bar{D}$. However, if $\bar{D}(t)=e^{-\lambda t} \delta^{t}+\left(1-e^{-\lambda t}\right) \beta \delta^{t}$ as in the text, then there is a solution $(c, V)$ in which $V(t, x)$ takes the form $e^{-\lambda t} \delta^{t} w(x)+\left(1-e^{-\lambda t}\right) \beta \delta^{t} v(x)$ iff $(c, v, w)$ satisfies the Bellman system of the PF consumer. In other words: any solution of the Bellman system of the PF consumer generates a solution of the Bellman system of the $\bar{D}$ consumer; but the possibility remains that the Bellman system of the $\bar{D}$ consumer has other solutions.

${ }^{16}$ For example, McClure et al (2007) estimate a $50 \%$ discount rate over the course of an hour for food/drink rewards. In most intertemporal choice studies, sharp short-run discounting (at least 10\% and usually much more) is observed at horizons of hours and days (e.g., see Ainslie 1992, Frederick, Loewenstein, and O'Donoghue 2002). 
a technique with precedents (e.g., trembling-hand perfect equilibrium and proper equilibrium). ${ }^{17}$ The second way is to derive the Bellman equation of the IG consumer directly from an analysis of her objective. This has the advantage that it generates intuitive insights into the logic of the IG model. We describe the first approach - taking the limit of the Bellman system of the PF consumer - here. For the alternative approach, see Online Appendix E.

Suppose the triple $\left(c_{\lambda}, v_{\lambda}, w_{\lambda}\right)$ solves the Bellman system of the PF consumer (for a given value of $\lambda$ ). In particular,

$$
\begin{aligned}
& 0=\frac{1}{2} \sigma^{2} x^{2} v_{\lambda}^{\prime \prime}+\left(\mu x+y-c_{\lambda}\right) v_{\lambda}^{\prime}-\gamma v_{\lambda}+u\left(c_{\lambda}\right), \\
& \frac{1}{\gamma} u(0) \leq v_{\lambda} \leq \bar{v}
\end{aligned}
$$

for all $x \in[0, \infty)$, and

$$
\left\{\begin{array}{ll}
u^{\prime}\left(c_{\lambda}\right)=w_{\lambda}^{\prime} & \text { if } x>0 \\
u^{\prime}\left(c_{\lambda}\right)=\max \left\{u^{\prime}(y), w_{\lambda}^{\prime}\right\} & \text { if } x=0
\end{array}\right\}
$$

Moving to a subsequence if necessary, suppose further that $v_{\lambda}$ and $w_{\lambda}$ (along with their first and second derivatives) and $c_{\lambda}$ converge to limiting functions $v$ and $w$ (along with their first and second derivatives) and $c .{ }^{18}$ Then the equations characterizing $(c, v, w)$ can be derived as follows.

Note first that equation (10) does not depend directly on $\lambda$. This equation only applies

\footnotetext{
${ }^{17}$ For example, in the case of trembling-hand perfection, one finds the set of all Nash equilibria of an $\varepsilon$-perturbed version of the original game; and then one finds the set of limit points of these equilibria as $\varepsilon$ goes to 0 . In the case of the IG model, one finds the set of all SMPE of the game with finite $\lambda$; and then one finds the set of limit points of these equilibria as $\lambda$ goes to $\infty$.

${ }^{18} \mathrm{~A}$ rigorous proof of the fact that $v_{\lambda}$ and $w_{\lambda}$ converge in this way is beyond the scope of the current paper. However, we offer the following motivation for the mathematically minded reader. First, it can be shown that $c$ is never infinite. Hence equations (5) and (6) are non-degenerate quasilinear elliptic differential equations in $(0, \infty)$. Second, inequalities (8) and (9) show that the sets $V=\left\{v_{\lambda} \mid \lambda \in[0, \infty)\right\}$ and $W=\left\{w_{\lambda} \mid \lambda \in[0, \infty)\right\}$ are equibounded. Third, combining the first and second observations, we see that $V$ and $W$ are precompact in the space $\mathcal{C}_{\text {loc }}^{2,1}((0, \infty))$ of functions which, along with their first and second derivatives, are Lipschitz continuous on compact subsets of $(0, \infty)$. Passing to a subsequence if necessary, we therefore conclude that $\exists v, w \in \mathcal{C}_{\text {loc }}^{2,1}((0, \infty))$ s.t. $v_{\lambda} \rightarrow v$ and $w_{\lambda} \rightarrow w\left(\right.$ both in $\left.\mathcal{C}_{\text {loc }}^{2,1}((0, \infty))\right)$ as $\lambda \rightarrow \infty$. Fourth, the convergence of $c_{\lambda}$ (which lies in $\left.\mathcal{C}_{\text {loc }}^{1,1}((0, \infty))\right)$ can be deduced from that of $w_{\lambda}^{\prime}$ (which likewise lies in $\mathcal{C}_{\text {loc }}^{1,1}((0, \infty))$ ) by inverting the first-order condition $u^{\prime}\left(c_{\lambda}\right)=w_{\lambda}^{\prime}$. We denote the limit of $c_{\lambda}$ by $c$. Fifth, it can be shown, that the appropriate boundary conditions (which do not involve second derivatives) also hold. Finally, we show below that the Bellman system of the IG consumer has a unique solution. Hence the limit point $(c, v, w)$ is unique. Hence, $\left(c_{\lambda}, v_{\lambda}, w_{\lambda}\right)$ converges (and not just along a suitably chosen subsequence).
} 
after the transition to the future has taken place, so it is not affected by the arrival rate of the future. Letting $\lambda \rightarrow \infty$ therefore preserves the form of (10), yielding

$$
0=\frac{1}{2} \sigma^{2} x^{2} v^{\prime \prime}+(\mu x+y-c) v^{\prime}-\gamma v+u(c)
$$

for all $x \in[0, \infty)$. In other words, just as $v_{\lambda}$ was the expected present discounted value obtained when consumption was chosen according to the exogenously given consumption function $c_{\lambda}$, so $v$ is the expected present discounted value obtained when consumption is chosen according to the exogenously given consumption function $c$.

Second, $w_{\lambda}-\beta v_{\lambda}$ is the value function of a consumer who: (i) has utility function $(1-\beta) u$; (ii) discounts the future exponentially at rate $\gamma+\lambda$; and (iii) has the exogenously given consumption function $c_{\lambda} \cdot{ }^{19}$ Hence $w_{\lambda}-\beta v_{\lambda} \rightarrow 0$ as $\lambda \rightarrow \infty .{ }^{20}$ In particular,

$$
w=\beta v
$$

for all $x \in[0, \infty)$.

Third, like equation (10), equation (12) does not depend directly on $\lambda$. Letting $\lambda \rightarrow \infty$ therefore preserves the form of this equation, yielding

$$
\left\{\begin{array}{ll}
u^{\prime}(c)=w^{\prime} & \text { if } x>0 \\
u^{\prime}(c)=\max \left\{u^{\prime}(y), w^{\prime}\right\} & \text { if } x=0
\end{array}\right\} .
$$

Just as $c_{\lambda}$ was the optimal consumption function when the current-value function was $w_{\lambda}$, so $c$ is the optimal consumption function when the current-value function is $w$.

Fourth, passing to the limit in equation (11), we obtain the global bounds

$$
\frac{1}{\gamma} u(0) \leq v \leq \bar{v}
$$

\footnotetext{
${ }^{19}$ Note that $w_{\lambda}$ is obtained by discounting back to time $s_{0}$ the utility flow $u\left(c_{\lambda}\right)$ during the span of control of self 0 , and the utility flow $\beta u\left(c_{\lambda}\right)$ during the spans of control of selves $1,2, \ldots$ Similarly, $v_{\lambda}$ is obtained by discounting back to time $s_{1}$ the utility flow $u\left(c_{\lambda}\right)$ during the span of control of self 1 , and the utility flow $u\left(c_{\lambda}\right)$ during the spans of control of selves $2,3, \ldots$ However, $v_{\lambda}$ could just as well be obtained by discounting back to time $s_{0}$ the utility flow $u\left(c_{\lambda}\right)$ during the span of control of self 0 , and the utility flow $u\left(c_{\lambda}\right)$ during the spans of control of selves $1,2, \ldots$. Subtracting $\beta$ times this latter utility flow from the utility flow for $w_{\lambda}$, we see that $w_{\lambda}-\beta v_{\lambda}$ is obtained by discounting back to time $s_{0}$ the utility flow $(1-\beta) u\left(c_{\lambda}\right)$ during the span of control of self 0 , and the utility flow 0 during the span of control of selves $1,2, \ldots$ That is, $w_{\lambda}-\beta v_{\lambda}$ is the value function described in the text.

${ }^{20}$ The assumption that $u$ is bounded below is used here. It implies that $w_{\lambda}-\beta v_{\lambda} \geq \frac{1-\beta}{\gamma+\lambda} u(0)$.
} 
for all $x \in[0, \infty)$. However, economic intuition suggests that the different selves should be able to overcome the coordination problem among themselves, at least to the extent of obtaining a minimum flow utility of $u(y)$ instead of $u(0)$. This intuition is vindicated by the following lemma, which allows us to replace (16) with the tighter

$$
\frac{1}{\gamma} u(y) \leq v \leq \bar{v}
$$

for all $x \in[0, \infty)$, and which is important for our theory.

Lemma 2. Suppose that $u$ is bounded below. Then $v \geq \frac{1}{\gamma} u(y)$.

Proof. See Appendix A.

Taken together, equations (13-15) and (17) constitute the Bellman system of the IG consumer. Eliminating $w$ from this system, we arrive at the following definition:

Definition 3. The Bellman equation of the IG consumer consists of the differential equation

$$
0=\frac{1}{2} \sigma^{2} x^{2} v^{\prime \prime}+(\mu x+y-c) v^{\prime}-\gamma v+u(c)
$$

for all $x \in[0, \infty)$, the optimality condition

$$
\left\{\begin{array}{ll}
u^{\prime}(c)=\beta v^{\prime} & \text { if } x>0 \\
u^{\prime}(c)=\max \left\{u^{\prime}(y), \beta v^{\prime}\right\} & \text { if } x=0
\end{array}\right\}
$$

and the global bounds

$$
\frac{1}{\gamma} u(y) \leq v \leq \bar{v}
$$

for all $x \in[0, \infty) .^{21}$

Notice that the Bellman equation of the IG consumer differs from the Bellman equation of an exponential consumer with utility function $u$ and discount rate $\gamma$ only in that the

\footnotetext{
${ }^{21}$ In order to give a mathematically complete definition, we need to say what we mean by a solution of the Bellman equation of the IG consumer. From a mathematical point of view, the best answer is 'a viscosity solution'. This is because the equation is degenerate, in the sense that the coefficient of $v^{\prime \prime}$ (namely $\frac{1}{2} \sigma^{2} x^{2}$ ) tends to 0 as $x$ tends to 0 . However, from a practical point of view, a perfectly satisfactory answer is 'a classical solution'. This is because: (i) any classical solution is a fortiori a viscosity solution; (ii) as Theorem 5 below shows, the Bellman equation of the IG consumer has a unique viscosity solution; and (iii) this unique viscosity solution is in fact twice continuously differentiable, and therefore a classical solution. For an introduction to viscosity solutions, and an explanation of their relationship to classical solutions, see Crandall et al (1992).
} 
marginal value of (future) wealth $v^{\prime}$ is multiplied by the factor $\beta$ in the optimality condition (19). Furthermore the former reduces to the latter if we put $\beta=1$. The presence of the multiplicative $\beta$ term is a slight variation on the usual form of the Envelope Theorem. This "new" Envelope Theorem is quite natural since the future arrives instantaneously, and the future has continuation value $\beta v$. Hence the marginal value of wealth is $\beta v^{\prime}$. This tells us two things. First, the liquidity constraint binds iff $x=0$ and $\beta v^{\prime}<u^{\prime}(y)$, in which case $c=y$ (or, equivalently, $u^{\prime}(c)=u^{\prime}(y)$ ). Second, if the liquidity constraint does not bind, then the current self sets consumption in such a way that $u^{\prime}(c)=\beta v^{\prime}$.

Notice too that, while both our characterization of equilibrium in the PF model in terms of the Bellman system of the PF consumer, and our derivation of the Bellman equation of the IG consumer from the Bellman system of the PF consumer, used the assumption that $u$ is bounded below, the Bellman equation of the IG consumer as such makes economic and mathematical sense whether or not $u$ is bounded below. ${ }^{22}$ This raises the question whether it is possible to derive the IG model when $u$ is not bounded below.

Note first that this question relates to the behavior of equilibria of the PF model when $\lambda$ is large. In the following discussion, we therefore restrict attention to $\lambda \in \Lambda=$ $[\max \{0, \mu-\gamma\}, \infty)$. Note second that, whether or not $u$ is bounded below, the PF model possesses equilibria in which $w_{\lambda}$ is bounded below. We call such equilibria "well-behaved". Furthermore, if $u$ is bounded below, then all equilibria are well-behaved. Third, in any well-behaved equilibrium, we have $w_{\lambda} \geq \underline{w}_{\lambda}=\frac{\gamma+\beta \lambda}{\gamma(\gamma+\lambda)} u(y)$. In other words, if $w_{\lambda}$ is bounded below, then it is bounded below by the specific quantity $\underline{w}_{\lambda}$. Fourth, $\underline{w}_{\lambda}$ is itself bounded below on $\Lambda$ (by $\left.\min \left\{\frac{1}{\gamma} u(y), \frac{\beta}{\gamma} u(y)\right\}\right)$.

Armed with this extra information, the discussion above adapts easily to show that, moving to a subsequence if necessary, any sequence of well-behaved solutions of the Bellman system of the PF consumer converges, as $\lambda \rightarrow \infty$, to a solution of the Bellman equation of the IG consumer.

The only catch is that, when $u$ is not bounded below, there exist equilibria for which $w_{\lambda}(0)=-\infty .^{23}$ We call such equilibria "pathological". For example, if $u$ is not bounded below, then the PF model admits a pathological equilibrium in which the consumption function is 0 . The mathematical logic behind this equilibrium is that, because future

\footnotetext{
${ }^{22}$ The global bounds (8-9) in the PF model involve $u(0)$, which is negatively infinite if $u$ is unbounded below, whereas the global bounds $(20)$ in the IG model involve $u(y)$, which is bounded since $y>0$.

${ }^{23}$ Note that $w_{\lambda}$ must be non-decreasing. This is because the current self has access to what is, effectively, a free-disposal technology: she can consume at an arbitrarily high rate. Hence the only way in which $w_{\lambda}$ can fail to be bounded below is if $w_{\lambda}(0)=-\infty$.
} 
selves consume at rate 0 , the continuation value of the current self will be $-\infty$, no matter what she does. Hence her current value will be $-\infty$, no matter what she does. Hence she may as well consume 0 too. This mathematical logic flies in the face of the intuitive logic of the situation: consuming nothing is the last thing that the current self should be doing, since by doing so she is passing on resources to selves who will never use them.

Overall, it makes sense to rule out the pathological equilibria and focus on the wellbehaved equilibria. These always exist. Moreover, moving to a subsequence if necessary, they converge to an equilibrium of the IG model. Finally, as we show below, equilibrium is unique in the IG model. Hence no subsequence is in fact necessary: the well-behaved equilibria converge to the equilibrium of the IG model.

\section{Existence, Uniqueness and Value-Function Equivalence}

In this section we show that the value function of the IG consumer exists and is unique. To prove this, we use a key intermediate result. We describe an alternative consumer with dynamically consistent preferences and a slightly altered utility function $\widehat{u}$. We show that a value function $v$ solves the Bellman equation of the IG consumer if and only if it solves the Bellman equation of this dynamically consistent " $\widehat{u}$ consumer". We call this result "value-function equivalence". We also emphasize that value-function equivalence is not the same as observational equivalence, and indeed that observational equivalence does not hold: the consumption function of the IG consumer is not the same as the consumption function of the $\widehat{u}$ consumer.

Value-function equivalence implies both the existence and the uniqueness of the value function of the IG consumer, for the simple reason that the $\widehat{u}$ consumer solves an optimization problem, and the value function of an optimization problem always exists and is unique. Uniqueness is the most important property of the IG model: the IG model resolves the multiplicity problem that has plagued the literature on dynamically inconsistent preferences. The current section also discusses a number of other issues, including the extension of the uniqueness result to the deterministic version of our model (in which asset returns are non-stochastic).

5.1. Assumptions. Before proceeding further, we introduce the simple assumptions on which the analysis in the rest of the paper will be based:

$$
\text { A1 } u(c)=\left\{\begin{array}{ll}
\frac{1}{1-\rho}\left(c^{1-\rho}-1\right) & \text { if } \rho \neq 1 \\
\ln (c) & \text { if } \rho=1
\end{array}\right\}
$$


A2 $1-\beta<\rho$

A3 $\mu<\bar{\mu}$, where $\bar{\mu}=\left\{\begin{array}{ll}\frac{1}{1-\rho} \gamma+\frac{1}{2} \rho \sigma^{2} & \text { if } \rho<1 \\ \infty & \text { if } \rho \geq 1\end{array}\right\}$.

Assumption A1 is standard: $u$ has constant relative risk aversion $\rho{ }^{24}$ Assumption A2 ensures that the dynamic inconsistency of the IG consumer (as measured by $1-\beta$ ) is less than the coefficient of relative risk aversion. ${ }^{25}$ This inequality would be satisfied in a standard calibration: empirical estimates of the coefficient of relative risk aversion $\rho$ typically lie between $\frac{1}{2}$ and 5 ; and the short-run discount factor $\beta$ is typically thought to lie between $\frac{1}{2}$ and $1 .{ }^{26}$ Assumption A3 is a one-sided (hence weaker) version of a standard integrability assumption. ${ }^{27}$ It ensures that the consumer's expected lifetime utility is not positively infinite even when the utility function is unbounded above (i.e. $\rho \leq 1$ ). It achieves this by ensuring that wealth does not grow too fast. ${ }^{28}$

In fact, the assumption of constant relative risk aversion is more restrictive than it needs to be (simplifying exposition). We could instead assume only that the utility function has bounded relative risk aversion and bounded relative prudence: in other words, relative risk aversion and relative prudence vary with consumption. This general case is analyzed in an earlier working paper (Harris and Laibson 2000), and the argument is summarized in Online Appendix H.

5.2. The Utility Function $\widehat{u}$. The new utility function $\widehat{u}$ depends not only on consumption $\widehat{c}$, but also on wealth $x$ : when $x>0, \widehat{u}(\widehat{c}, x)=\widehat{u}_{+}(\widehat{c}) ;$ but when $x=0$, $\widehat{u}(\widehat{c}, x)=\widehat{u}_{0}(\widehat{c})$. In other words, the wealth-dependent utility function $\widehat{u}$ is made up of two wealth-independent utility functions $\widehat{u}_{+}:(0, \infty) \rightarrow \mathbb{R}$ and $\widehat{u}_{0}:(0, y] \rightarrow \mathbb{R}$, with $\widehat{u}_{+}$

\footnotetext{
${ }^{24}$ Notice that Assumptions A1-A3 do not imply that $u$ is bounded below. This is because the analysis which follows takes the Bellman equation of the IG consumer (namely (18-20)) as its starting point, and neither this equation nor our analysis of it depends on $u$ being bounded below.

${ }^{25}$ The case $1-\beta>\rho$ can also be analyzed. Now the consumer's desire to consume immediately (as measured by $1-\beta$ ) outweighs her desire to smooth consumption (as measured by $\rho$ ). The current self therefore consumes all her financial wealth during her instant of control, forcing all subsequent selves to consume only their labor income $y$. Since $\frac{u(c)}{c} \rightarrow 0$ as $c \rightarrow \infty$, this burst of consumption by the current self contributes nothing to the integral of lifetime utility. The value function is therefore $v=\frac{1}{\gamma} u(y)$. (Notice that $v^{\prime}=0$. This is consistent with the infinite consumption rate.)

${ }^{26}$ See Laibson et al (1998) and Ainslie (1992).

${ }^{27}$ In the model with $y=0$ and $\beta=1$, it is standard to assume $\gamma>(1-\rho)\left(\mu-\frac{1}{2} \rho \sigma^{2}\right)$. This can be divided into two parts: $\mu<\frac{1}{1-\rho} \gamma+\frac{1}{2} \rho \sigma^{2}$ if $\rho<1$; and $\mu>\frac{1}{1-\rho} \gamma+\frac{1}{2} \rho \sigma^{2}$ if $\rho>1$. Wealth must not rise too fast when $u$ is unbounded above; and must not fall too fast when $u$ is unbounded below.

${ }^{28}$ We do not need the other side of the standard assumption, which is designed to ensure that expected lifetime utility is not negatively infinite, because we assume $y>0$.
} 
being used to evaluate consumption when wealth is positive, and $\widehat{u}_{0}$ being used to evaluate consumption when wealth is zero. The utility function $\widehat{u}_{+}$is simply a rescaling of $u$. It is given by the formula

$$
\widehat{u}_{+}(\widehat{c})=\frac{\psi}{\beta} u\left(\frac{1}{\psi} \widehat{c}\right)+\frac{\psi-1}{\beta} \text { for } \widehat{c} \in(0, \infty)
$$

where

$$
\psi=\frac{\rho-(1-\beta)}{\rho} .
$$

(Notice that, under our assumptions, $\psi \in(0,1)$.) The utility function $\widehat{u}_{0}$ coincides with $\widehat{u}_{+}$for $\widehat{c} \in(0, \psi y)$, and with the tangent to $\widehat{u}_{+}$at $\psi y$ for $\widehat{c} \in[\psi y, y]{ }^{29}$

The relationship between $u, \widehat{u}_{+}$and $\widehat{u}_{0}$ is plotted in Figure 4, using the parameter values $\beta=\frac{2}{3}, \rho=\frac{3}{4}$ and $y=1$. Notice that $\widehat{u}_{+}(\widehat{c})<u(\widehat{c})$ for all $\widehat{c} \in(0, \infty)$ and $\widehat{u}_{0}(\widehat{c})<u(\widehat{c})$ for all $\widehat{c} \in(0, y)$. This makes sense: the $\widehat{u}$ consumer optimizes fully, while the IG consumer does not. Hence the $\widehat{u}$ consumer must be suitably handicapped in order to prevent her from achieving a higher value than the IG consumer. Notice too that $\widehat{u}_{0}(y)=u(y)$. Once again this makes sense: in the liquidity constrained case, both the $\widehat{u}$ consumer and the IG consumer consume their labor income $y$ forever. So we must have $\widehat{u}_{0}(y)=u(y)$ if they are both to obtain the same value.

5.3. Value-Function Equivalence. The $\widehat{u}$ consumer is a consumer who: (i) has the utility function $\widehat{u}$; (ii) faces the same wealth dynamics as the IG consumer; and (iii) discounts the future exponentially at rate $\gamma$.

Theorem 4 [Value-Function Equivalence]. $v$ is a value function of the IG consumer iff $v$ is a value function of the $\widehat{u}$ consumer.

Proof. See Appendix B.

The $\widehat{u}$ consumer has both conventional and unconventional features. On the conventional side, she discounts exponentially (at rate $\gamma$ ), so she has dynamically consistent preferences. On the unconventional side, her utility function depends on her financial wealth $x$. When $x>0$, her utility function is $\widehat{u}_{+}$. When $x=0$, it is $\widehat{u}_{0}$. This unconventional wealth-dependence is needed to generate value-function equivalence.

Using the Value-Function Equivalence Theorem, we can reduce the study of the problem of the IG consumer, which is game-theoretic, to the study of the problem of the $\widehat{u}$

\footnotetext{
${ }^{29}$ I.e. $\widehat{u}_{0}(\widehat{c})=\widehat{u}_{+}(\widehat{c})$ for $\widehat{c} \in(0, \psi y]$, and $\widehat{u}_{0}(\widehat{c})=\widehat{u}_{+}(\psi y)+(\widehat{c}-\psi y) \widehat{u}_{+}^{\prime}(\psi y)$ for $\widehat{c} \in[\psi y, y]$.
} 
consumer, which is decision-theoretic (i.e. non-strategic). There is, however, an important caveat: while the value function of the IG consumer coincides with value function of the $\widehat{u}$ consumer, it is not the case that the consumption function of the IG consumer coincides with the consumption function of the $\widehat{u}$ consumer. In particular, value-function equivalence does not translate into observational equivalence in behavior. ${ }^{30}$

From the Value-Function Equivalence Theorem, it is easy to deduce the existence and uniqueness of equilibrium in the IG model:

Theorem 5 [Existence and Uniqueness]. The IG model has a unique equilibrium.

The intuition for this result follows. First, optimization problems have unique value functions, since there cannot be two state-contingent values that are both best values. Hence, the $\widehat{u}$ consumer - who is an optimizer - must have a unique value function. Second, the set of possible value functions of the $\widehat{u}$ consumer coincides with the set of possible solutions of her Bellman equation. Hence the Bellman equation of the $\widehat{u}$ consumer has a unique solution, namely her value function. Third, the Bellman equation of the IG consumer is identical to the Bellman equation of the $\widehat{u}$ consumer. Hence the Bellman equation of the IG consumer likewise has a unique solution, and this solution is the value function of the $\widehat{u}$ consumer. Finally, one can use this common value function to derive both the equilibrium policy function of the IG consumer, which is unique, and the optimal policy function of the $\widehat{u}$ consumer, which is different from that of the IG consumer, and which is not necessarily unique. ${ }^{31}$

Proof. See Appendix C.

5.4. The Deterministic Case: A Refinement. Until now we have assumed that the standard deviation of asset returns is strictly positive $(\sigma>0)$. In other words, we have been studying the stochastic IG model. In the present subsection, we discuss the deterministic IG model $(\sigma=0)$.

\footnotetext{
${ }^{30}$ For a detailed exploration of the relationship between $c$ and $\widehat{c}$, see footnote 31 below and Online Appendix G.7.

${ }^{31}$ For the IG consumer, equilibrium consumption is generated by the first-order condition $u^{\prime}(c)=\beta v^{\prime}$ if $x>0$ and $u^{\prime}(c)=\max \left\{u^{\prime}(y), \beta v^{\prime}\right\}$ if $x=0$; and for the $\widehat{u}$ consumer, optimal consumption is generated by the first-order condition $\widehat{u}_{+}^{\prime}(\widehat{c})=v^{\prime}$ if $x>0$ and $\widehat{u}_{0}^{\prime}(\widehat{c})=\max \left\{\widehat{u}_{0}^{\prime}(y), v^{\prime}\right\}$ if $x=0$. Moreover, it follows directly from the definition of $\widehat{u}_{+}$that $\widehat{u}_{+}^{\prime}(\widehat{c})=\frac{1}{\beta} u^{\prime}\left(\frac{1}{\psi} \widehat{c}\right)$. Hence, when $x>0$, we have $u^{\prime}(c)=\beta v^{\prime}=\beta \widehat{u}_{+}^{\prime}(\widehat{c})=u^{\prime}\left(\frac{1}{\psi} \widehat{c}\right)$ or $\widehat{c}=\psi c$. In particular, $\widehat{c}$ is different from $c$. Furthermore, if $v^{\prime}(0)=\widehat{u}_{0}^{\prime}(y)$ (a situation that arises iff $\mu=\mu_{1}$ ), then $\widehat{c}$ can take on any value in the interval $[\psi y, y]$ and is not therefore unique.
} 
We begin by defining the Bellman equation of the deterministic IG consumer. We then note that - as in the stochastic case - the Bellman equation of the deterministic IG consumer is identical to the Bellman equation of the deterministic $\widehat{u}$ consumer. The Value-Function Equivalence Theorem therefore holds for the deterministic IG model. In particular, the deterministic IG model has a unique value function. This result is significant, because it provides a way of resolving concerns that deterministic hyperbolic models may have a continuum of equilibria (cf. Krusell and Smith (2000), Ekeland and Lazrak (2006, 2010), and Karp (2007)). ${ }^{32}$ It eliminates not just the possibility of a continuum of value functions, but even the possibility a finite multiplicity of value functions.

Next, in order to unify our deterministic and stochastic results, we show that the value function of the deterministic IG consumer is the limit of the value function of the stochastic IG consumer as noise converges to zero $(\sigma \downarrow 0)$. This implies that the value function of the deterministic IG consumer is precisely the value function that would be selected by a 'trembling-hand' analysis. ${ }^{33}$ In other words, uniqueness in the deterministic IG model can be understood as a refinement result.

Finally, the deterministic model is tractable: the Bellman equation of the deterministic IG consumer can be transformed into an autonomous first-order differential equation, whereas the Bellman equation of the stochastic IG consumer is a second-order nonautonomous differential equation. An earlier draft of this paper, Harris and Laibson (2000), expands on these points and provides a complete characterization of the value and policy functions of the deterministic case.

Turning now to the details, the first step is to define the Bellman equation of the deterministic IG consumer. In Definition 3 above, we defined the Bellman equation of the IG consumer for the case $\sigma>0$. Definition 3 can therefore be thought of as the definition of the Bellman equation of the stochastic IG consumer. We define the Bellman equation

\footnotetext{
${ }^{32}$ Like our paper, these papers are all continuous-time models and they all study discount functions that are not exponential. However, there are some differences: (i) their models are embedded in a deterministic economy and ours is in a stochastic economy (stochasticity smooths the value function and is also critical for our characterization and global uniqueness results); (ii) our model sets up a present-future dichotomy with a stochastic transition from one to the other (the stochastic transition makes the problem stationary, improving tractability); (iii) finally, these papers emphasize that multiple equilibria exist, whereas our key result is to show how to set up the model to obtain a unique equilibrium.

${ }^{33}$ There is a close analogy between using the limit as $\sigma \downarrow 0$ to identify a unique equilibrium of the deterministic IG model and using trembling-hand perfection to refine the set of Nash equilibria of a finite game. In our case, the stochasticity of asset returns ensures that, starting from any interior state, every other interior state will be reached with positive probability. In the case of trembling-hand perfection, trembles ensure that all successor nodes of the game are reached with positive probability.
} 
of the deterministic IG consumer by putting $\sigma=0$ everywhere in Definition $3 .^{34}$

The second step is to demonstrate that value-function equivalence holds: ${ }^{35}$

Theorem 6 [Value-Function Equivalence]. $v$ is a value function of the deterministic IG consumer iff $v$ is a value function of the deterministic $\widehat{u}$ consumer.

From this in turn we obtain uniqueness:

Theorem 7 [Existence and Uniqueness]. The deterministic IG model has a unique value function.

The fourth step is to unify our deterministic and stochastic results:

Theorem 8. For all $\sigma>0$, let $v_{\sigma}$ be the value function of the stochastic IG consumer; and let $v$ be the value function of the deterministic IG consumer. Then $v_{\sigma} \rightarrow v$ uniformly on compact subsets of $[0, \infty)$ as $\sigma \downarrow 0$.

Proof. See Appendix D.

Remark. Assumptions A1 and A2 do not involve the parameter $\sigma$, and Assumption A3 becomes more restrictive as $\sigma$ decreases. For the analysis of this section, it therefore suffices to use the special case of Assumption A3 in which $\sigma=0$.

\section{The Consumption Function when $y=0$}

In the current section, we begin the analysis of the consumption function by studying the limit cases of the IG model and the PF model in which there is no labor income. ${ }^{36}$ The case $y=0$ is useful for three reasons. First, both the IG model (in which $\lambda \rightarrow \infty$ and the present lasts only an instant) and the PF model (in which $\lambda<\infty$ and the present has strictly positive duration) become highly tractable when $y=0$ : the value and policy functions corresponding to the unique equilibrium are linear in wealth; and closed-form

\footnotetext{
${ }^{34}$ As in the case of the Bellman equation of the stochastic IG consumer, we need to say what we mean by a solution of the Bellman equation of the deterministic IG consumer. This time there is only one possible answer: 'a viscosity solution'. This is because, for $\mu \in\left(\gamma, \frac{1}{\beta} \gamma\right)$, the unique solution of the equation has a convex kink. In particular, it is not a classical solution. For an introduction to viscosity solutions, and an explanation of their relationship to classical solutions, see Crandall et al (1992).

${ }^{35}$ Following math convention, we use a solid box, $\mathbf{\square}$, to signify that a theorem is stated without proof.

${ }^{36}$ The paper focuses on the case $y>0$. The case $y=0$ is analyzed only in the present section. The two cases involve different considerations, and are treated separately.
} 
expressions can be found for both functions. Second, models in which policy functions are linear in wealth are often used as benchmarks in the economics literature (for example, Merton 1971, Barro 1999, Luttmer and Marriotti 2003). Third, we can use the case $y=0$ to study the relationship between the IG model and the PF model. Empirical evidence suggests that present bias affects utility flows in the immediate present (i.e., the current week, day, or even hour) and does not affect utility flows that will come a few weeks or months from now (e.g., McClure et al 2007). Hence, present bias describes a person who wishes to break their diet today but keep the diet starting tomorrow, or a student who prefers to take today off but get back to work tomorrow. Hence, present bias applies for $\lambda \simeq 365$. Using the case $y=0$, we show that $\lambda \rightarrow \infty$ is a good approximation for all $\lambda$ values in the interval $[12, \infty)$, which subsumes the empirically relevant interval $[365, \infty)$.

6.1. The PF Model with $y=0$. To analyze the PF model with $y=0$, we make two assumptions that differ from the $y>0$ case. First, we focus exclusively on equilibria in linear consumption functions. This allows us to dispense with the global upper and lower bounds. Second, we need to strengthen Assumption A3 (which requires that $\mu<$ $\frac{1}{1-\rho} \gamma+\frac{1}{2} \rho \sigma^{2}$ when $\rho<1$ ) by requiring in addition that $\mu>\frac{1}{1-\rho} \gamma+\frac{1}{2} \rho \sigma^{2}$ when $\rho>1 .^{37}$

The Bellman system of the PF model with $y=0$ is simpler than that of the PF consumer with $y>0$. It consists of the pair of differential equations

$$
\begin{aligned}
& 0=\frac{1}{2} \sigma^{2} x^{2} v^{\prime \prime}+(\mu x-c) v^{\prime}-\gamma v+u(c) \\
& 0=\frac{1}{2} \sigma^{2} x^{2} w^{\prime \prime}+(\mu x-c) w^{\prime}+\lambda(\beta v-w)-\gamma w+u(c)
\end{aligned}
$$

for all $x>0$, and the optimality condition

$$
u^{\prime}(c)=w^{\prime}
$$

again for all $x>0$. In particular, we no longer require that the differential equations or the optimality conditions hold at $x=0$, and we no longer require the global bounds.

It is natural to look for a solution to equations (21-23) in the form

$$
v(x)=\Theta u(\theta x), \quad w(x)=\Phi u(\phi x), \quad c(x)=\alpha x,
$$

where the constants $\Theta, \Phi, \theta, \phi$ and $\alpha$ are all required to be strictly positive. Making this

\footnotetext{
${ }^{37} \mathrm{Cf}$. footnotes 27 and 28 .
} 
substitution leads to the following quadratic equation for $\alpha$ :

$$
0=\frac{\lambda}{1+\lambda}((\rho+\beta-1) \alpha-\widetilde{\gamma})+\frac{1}{1+\lambda}\left(\rho(1-\rho) \alpha^{2}+(2 \rho-1) \widetilde{\gamma} \alpha-\widetilde{\gamma}^{2}\right)
$$

where

$$
\widetilde{\gamma}=\gamma-(1-\rho)\left(\mu-\frac{1}{2} \rho \sigma^{2}\right)
$$

See Online Appendix F for details. Furthermore it can be shown that only one of the two solutions of this quadratic is relevant. This solution is always positive, varying from $\frac{\widetilde{\gamma}}{\rho}$ when $\lambda=0$ to $\frac{\widetilde{\gamma}}{\rho-(1-\beta)}$ when $\lambda=\infty .{ }^{38}$

A more concrete understanding of this solution, and especially of its behavior as $\lambda \rightarrow$ $\infty$, can be obtained by taking expansions in $\lambda^{-1}$. Indeed, we have

$$
\alpha=\frac{\tilde{\gamma}}{\rho+\beta-1}-\frac{(1-\beta) \beta \tilde{\gamma}^{2}}{(\rho+\beta-1)^{3}} \lambda^{-1}+\mathrm{O}\left(\lambda^{-2}\right) .
$$

The first-order effect of increasing $\lambda$ is therefore to increase the average propensity to consume. A higher value of $\lambda$ implies that the multiplicative $\beta$-discounting associated with the passage to the future arrives more quickly. More discounting lowers the value of future consumption, thereby raising the propensity to consume today.

6.2. The IG Model with $y=0$. To analyze the IG model with $y=0$ we make two assumptions that differ from the $y>0$ case (mirroring our approach with the PF model). First, we focus on equilibria in linear consumption functions. Second, we need to strengthen Assumption A3 by requiring that $\mu>\frac{1}{1-\rho} \gamma+\frac{1}{2} \rho \sigma^{2}$ when $\rho>1$.

The Bellman equation of the IG consumer with $y=0$ consists of the equation

$$
0=\frac{1}{2} \sigma^{2} x^{2} v^{\prime \prime}+(\mu x-c) v^{\prime}-\gamma v+u(c)
$$

and the optimality condition

$$
u^{\prime}(c)=\beta v^{\prime}
$$

both for all $x>0$.

This equation can be solved using the same methods as in Section 6.1. We look for

$$
v(x)=\Theta u(\theta x), \quad c(x)=\alpha x,
$$

\footnotetext{
${ }^{38}$ Assumption A2 implies that $\rho-(1-\beta)>0$, and Assumption A3 implies that $\widetilde{\gamma}>0$.
} 
where the constants $\Theta, \theta$ and $\alpha$ are all required to be strictly positive. Making this substitution leads to the conclusion that

$$
\alpha=\frac{\widetilde{\gamma}}{\rho+\beta-1},
$$

where $\widetilde{\gamma}=\gamma-(1-\rho)\left(\mu-\frac{1}{2} \rho \sigma^{2}\right)$ as before. ${ }^{39}$

It is easy to see that the right-hand side of equation (27) is the limit of the relevant solution of equation (24) as $\lambda \rightarrow \infty$. Hence the policy function of the PF model converges, as $\lambda \rightarrow \infty$, to the policy function of the IG model. It can also be shown, as one would expect in the light of the convergence of the policy functions, that the value function of the PF model converges to the value function of the IG model as $\lambda \rightarrow \infty$.

Barro (1999) and Luttmer and Mariotti (2003) analyze continuous-time economies with a general class of dynamically-inconsistent time preferences. In the economic environments studied in these two papers linear policy rules always support an equilibrium and the authors restrict attention to equilibria in this class.

Barro's deterministic economy has returns that vary over time due to aggregate growth dynamics, whereas our stochastic economy has returns that are i.i.d. For the log utility case $(\rho=1)$, the propensity to consume in our economy is $\alpha=\frac{\gamma}{\beta}$, which matches the propensity to consume that Barro derives for log utility when converging to the continuous time analog of the quasi-hyperbolic discount function.

Like Barro, Luttmer and Mariotti (2003) study a range of time preferences, including the continuous-time analog of the quasi-hyperbolic discount function. Unlike Barro, Luttmer and Mariotti study a stochastic endowment economy, which they use to characterize asset prices.

The IG model differs from Barro (1999) and Luttmer and Mariotti (2003) in that our analysis is valid whether the equilibrium policies are linear or non-linear. For example, the IG model can handle liquidity constraints, other forms of market incompleteness and utility functions outside of the constant relative risk aversion class. In addition, we obtain uniqueness for both the $y=0$ and $y>0$ versions of the IG model without restricting the class of policy functions.

6.3. Calibration of the $y=0$ Cases. We now provide a calibration of the $y=0$ models. For this calibration we fix the parameters $\gamma=0.05, \beta=\frac{2}{3}, \sigma=0.17$ and

\footnotetext{
${ }^{39}$ We also obtain $\Theta=\frac{1}{\gamma}$ and $\theta^{1-\rho}=\frac{\gamma}{\tilde{\gamma}+(1-\rho) \alpha}$.
} 
$\mu=0.06 .{ }^{40}$ We then vary the value of risk aversion $(\rho)$ and the hazard rate at which the future arrives $(\lambda)$. This calibration identifies the range of $\lambda$ for which the $y=0$ cases of the PF model and the IG model have quantitatively similar policy functions.

Specifically, we calculate the marginal propensity to consume for the PF model for $\lambda \in\left\{0, \frac{1}{10}, 1,12,52,365, \infty\right\}$. At $\lambda=0$, the future never arrives; at $\lambda=52$, the future arrives on average once a week; and at $\lambda=\infty$, the future arrives instantaneously. We believe that the appropriate calibration is $\lambda=365$, implying that the psychological future arrives on average once per day.

\begin{tabular}{c|cccc} 
& $\rho=\frac{1}{2}$ & $\rho=1$ & $\rho=2$ & $\rho=5$ \\
\hline$\lambda=0$ & 0.0472 & 0.0500 & 0.0405 & 0.000200 \\
$\lambda=\frac{1}{10}$ & 0.0797 & 0.0643 & 0.0463 & 0.000214 \\
$\lambda=1$ & 0.123 & 0.0733 & 0.0484 & 0.000214 \\
$\lambda=12$ & 0.140 & 0.0748 & 0.0486 & 0.000214 \\
$\lambda=52$ & 0.141 & 0.0750 & 0.0487 & 0.000214 \\
$\lambda=365$ & 0.142 & 0.0750 & 0.0487 & 0.000214 \\
$\lambda=\infty$ & 0.142 & 0.0750 & 0.0487 & 0.000214
\end{tabular}

Table 1: The marginal propensity to consume as a function of the coefficient of relative risk aversion $(\rho)$ and the arrival rate of the future $(\lambda)$.

Recall that the IG model is the case $\lambda \rightarrow \infty$. Table 1 shows that the IG model is a good approximation for the PF model as long as $\lambda \geq 12$. In other words, the IG model is a good approximation of the PF model as long as the present lasts on average about one month or less.

\section{The Consumption Function when $y>0$}

We now turn to the more challenging case with non-zero labor income. For this case, the PF model is not analytically tractable, and we therefore focus exclusively on the IG model. Three general properties emerge. We first provide an overview of these properties before delving into the details.

First, the consumption function is continuously differentiable in the interior of the wealth space. This is a consequence of Brownian motion in the wealth process. Brownian

\footnotetext{
${ }^{40}$ These are standard calibration values based either on historical data $(\sigma, \mu)$ or parameter estimates $(\gamma, \beta)$. See Laibson, Repetto, and Tobacman (2011).
} 
motion makes the value function twice continuously differentiable, and thereby eliminates discontinuities in the consumption function. More formally, optimality implies that $u^{\prime}(c)=\beta v^{\prime}$ when $x>0$. Differentiating this expression yields $u^{\prime \prime}(c) c^{\prime}=\beta v^{\prime \prime}$. Twice continuous differentiability of the value function therefore implies continuous differentiability of the consumption function when $x>0 .{ }^{41}$

Second, if the expected rate of return $\mu$ is low enough, the consumption function will have an upward discontinuity when wealth $x=0$. Intuitively, if $\mu$ is low, then the liquidity constraint binds at $x=0$; but, even when $\mu$ is low, it cannot bind at any strictly positive $x$ (no matter how small) since $x$ is a stock and $c$ is a flow. The sudden arrival of a binding liquidity constraint as $x$ falls from any strictly positive value to 0 causes a downward jump in $c$ from $c(0+)=\bar{c}>y$ to $c(0)=y$. Moreover this downward jump can be understood in terms of the consumer's propensity to value immediate rewards discretely more than delayed rewards. It does not arise when $\mu$ is sufficiently high, since in that case the liquidity constraint does not bind at $x=0$.

Third, it can happen that there is an interval over which the consumption function is downward sloping. This occurs if the expected rate of return $\mu$ takes on intermediate values. However, this non-monotonicity disappears when a bond is introduced, and the investor can take both long and short positions in the bond. We therefore view the first two properties as robust implications of the IG model, and the third property as an artefact of the bond-free model that we study in the present paper.

These properties of the IG model contrast with the properties of the continuous-time exponential model, the consumption function of which is continuous everywhere, including at $x=0$ where the liquidity constraint starts to bind, and monotonic for all choices of $\mu$. The properties of the IG model also contrast with the properties of the discrete-time quasihyperbolic model, the consumption function of which may have several downward sloping regions and a countable number of downward jumps. ${ }^{42}$ (Cf. Morris and Postlewaite 1997, Krusell and Smith 2000, Harris and Laibson 2001, Morris 2002.)

7.1. Comparative Statics on $\mu$. In order to simplify our description of the behavior of the consumption function, we vary the expected rate of return $\mu$ and hold the other

\footnotetext{
${ }^{41} \mathrm{~A}$ deeper analysis shows that the value function is actually infinitely differentiable on the whole wealth space (including the boundary). However, if the liquidity constraint binds, then this only translates into infinite differentiability of the consumption function in the interior of the wealth space, because in this case the consumption function is discontinuous at 0 .

${ }^{42}$ Downward sloping regions and jumps are not eliminated by adding a bond to the discrete-time model.
} 
parameters fixed. It turns out that there are three qualitative cases to consider.

Recall that Assumption A3 requires that $\mu<\bar{\mu}$, where $\bar{\mu}=\frac{1}{1-\rho} \gamma+\frac{1}{2} \rho \sigma^{2}$ if $\rho<1$ and $\bar{\mu}=\infty$ if $\rho \geq 1$. We will show that there exists $\mu_{1} \in(\gamma, \bar{\mu})$ such that the form of the consumption function depends on the interval in which $\mu$ lies: $(-\infty, \gamma),\left(\gamma, \mu_{1}\right)$ or $\left(\mu_{1}, \bar{\mu}\right)$. We refer to these cases as the low- $\mu$, intermediate- $\mu$ and high- $\mu$ cases respectively.

In all three cases, the consumption function is continuous everywhere except possibly when $x=0$, at which point the liquidity constraint may bind. When $\mu$ is low, the consumption function is everywhere increasing, but the liquidity constraint is binding, which generates an upward discontinuity at $x=0$. When $\mu$ is intermediate, there is an upward discontinuity at $x=0$, followed first by a downward sloping region and thereafter by an upward sloping region. When $\mu$ is high, the incentive to save is strong enough to make the consumption function globally continuous and increasing.

Figure 5 shows three consumption functions corresponding to the three cases for $\mu$. These functions were obtained from careful numerical simulations of our model, but we are also able to confirm their qualitative properties analytically. (See Online Appendix G.) All three functions use the parameter values $\beta=\frac{2}{3}, \gamma=0.05, \sigma=0.17, \rho=\frac{3}{4}$ and $y=1$. These values are illustrative, but they are all empirically sensible (and $y=1$ is a normalization): they involve a present bias of about a third, a long-run discount rate of $5 \%$, an annual standard deviation of stock returns of $17 \%$ and a coefficient of relative risk aversion of $\frac{3}{4}$. The differences between the functions are the result of varying $\mu$ over the set $\{0.04,0.07,0.09\}$ : the top consumption function corresponds to $\mu=0.04$ (a low- $\mu$ case); the middle consumption function corresponds to $\mu=0.07$ (an intermediate- $\mu$ case); and the bottom consumption function corresponds to $\mu=0.09$ (a high- $\mu$ case). We will refer back to this figure as we work through our formal results.

7.2. The low- $\mu$ case (discontinuity at zero wealth). The most novel case of our model is $\mu<\gamma$. The expected returns on the asset are not sufficiently attractive to induce the IG consumer to save when her wealth is zero, and the liquidity constraint binds. More precisely, let $\bar{c} \in(y, \infty)$ be the unique solution of

$$
u^{\prime}(\bar{c})=\beta \frac{u(\bar{c})-u(y)}{\bar{c}-y} .
$$

Theorem 9. If $\mu \in(-\infty, \gamma)$ then: $c(0)=y ; c(0+)=\bar{c}>y$; and $c^{\prime}>0$ on $(0, \infty)$. 
In other words: when the IG consumer has no wealth, she consumes all of her labor income; if she acquires even a little wealth, then her consumption jumps up from $y$ to $\bar{c}$; and her consumption increases monotonically with further increases in her wealth. In particular, her consumption function is strictly increasing.

Proof. See Online Appendix G.

To understand Equation (28) consider a consumer with strictly positive wealth. In the low $\mu$ case, the dynamics of wealth and consumption are causing wealth to trend lower. We refer to the (stochastic) moment at which wealth runs out as the 'crunch'. Suppose that the consumption level of the pre-crunch self is $\bar{c}$. Then the cost to the pre-crunch self of putting aside an extra $d x$ units of wealth is $u^{\prime}(\bar{c}) d x$. If the post-crunch self receives a windfall consisting of an extra $d x$ units of wealth, she can raise her consumption level from $y$ to $\bar{c}$ for a length of time $d t=d x /(\bar{c}-y)$. The benefit to the post-crunch self of this increase in consumption is $(u(\bar{c})-u(y)) d t$, and the benefit to the pre-crunch self is $\beta(u(\bar{c})-u(y)) d t$. The pre-crunch self is therefore indifferent between putting aside the extra $d x$ units of wealth and not putting them aside if and only if

$$
u^{\prime}(\bar{c}) d x=\beta(u(\bar{c})-u(y)) d t
$$

Substituting for $d t$ and dividing through by $d x$, we obtain equation (28).

As Theorem 9 implies, for the top consumption function in Figure 5: the liquidity constraint is binding, i.e. $c(0)=y=1$; there is an upward jump in consumption at $x=0$, from $c(0)=1$ to $c(0+)=\bar{c} \approx 3.45$; and consumption rises monotonically thereafter.

7.3. The high- $\mu$ case. The other polar case of our model is that in which $\mu>\mu_{1}$. In this case, the expected return is sufficiently attractive to induce the IG consumer to save even when her wealth is zero. More precisely:

Theorem 10. If $\mu \in\left(\mu_{1}, \infty\right)$ then: $c(0)<y$; $c(0+)=c(0)$; and $c^{\prime}>0$ on $[0, \infty)$.

In other words: when $x=0$, she consume strictly less than her labor income; acquiring a little wealth does not lead to a jump in consumption; and consumption increases strictly with $x$. The bottom consumption function in Figure 5 reflects this.

Proof. See Online Appendix G.

7.4. The intermediate- $\mu$ case. The remaining case of our model is that in which $\gamma<\mu<\mu_{1}$. Loosely speaking: when wealth is low, this case looks like the low- $\mu$ case; and 
when wealth is high, it looks like the high- $\mu$ case. However, the most striking feature is the behavior of the consumption function during the transition between the two regimes.

Theorem 11. If $\mu \in\left(\gamma, \mu_{1}\right)$ then: $c(0)=y ; c(0+)=\bar{c}>y$; and there exists $\bar{x} \in(0, \infty)$ such that $c^{\prime}<0$ on $(0, \bar{x})$ and $c^{\prime}>0$ on $(\bar{x}, \infty)$.

In other words, when the IG consumer has no wealth, she consumes all of her labor income. If she acquires even a little wealth, then her consumption jumps up from $y$ to $\bar{c}$. As her wealth increases from 0 to $\bar{x}$, her consumption decreases, but, once her wealth reaches $\bar{x}$, her consumption increases steadily with further increases in her wealth.

Proof. See Online Appendix G.

As Theorem 11 leads us to expect, for the middle consumption function in Figure 5, the liquidity constraint is binding, i.e. $c(0)=y=1$. There is also an upward jump in consumption at $x=0$, from $c(0)=1$ to $c(0+)=\bar{c} \approx 3.45$. Finally, consumption declines smoothly after the upward jump before bottoming out and rising thereafter.

Comparing Theorem 11 with Theorems 9 and 10, a simple pattern emerges. The strategic interaction between the current self and future selves induces a form of positive feedback: the higher the marginal propensity to save of tomorrow's self, the greater the willingness of the current self to save, and therefore the higher her own marginal propensity to save.

There are therefore two possible regimes: a high-consumption regime and a lowconsumption regime. When $\mu$ is low, the consumer finds herself in the high-consumption regime irrespective of her wealth. When $\mu$ is intermediate, the consumer finds herself in the high-consumption regime when her wealth is low, and in the low-consumption regime when her wealth is high. So, naturally, her consumption needs to decrease as her wealth increases in order to effect the transition between the two regimes. Finally, when $\mu$ is high, the consumer finds herself in the low-consumption regime irrespective of her wealth.

The non-monotonic consumption function in the intermediate- $\mu$ case is not a robust feature of our model. We can show that this non-monotonicity vanishes when we introduce a risk-free bond into the economy and allow investors to take long or short positions in the bond. Taking a large short position in the bond enables the consumer to take gambles that globally concavify her value function. This eliminates the regions of nonmonotonicity of the consumption function, since the value function would now have a slope that is monotonically falling in wealth, and the consumer equates her marginal utility of consumption to $\beta$ times the slope of her value function. 
Hence, two robust properties emerge from our analysis: a continuously differentiable consumption function in the interior of the wealth space, and the potential for an upward discontinuity of the consumption function at the point where the liquidity constraint binds $(x=0)$. This latter property cannot arise with an exponential discount function.

\section{Conclusions}

We have described a continuous-time model of quasi-hyperbolic discounting that extends the analysis of Barro (1999) and Luttmer and Mariotti (2003). Unlike these models, our instantaneous-gratification model allows for a generic class of preferences, includes liquidity constraints and places no restrictions on equilibrium policy functions. In our model, equilibrium is unique, resolving multiplicity problems in quasi-hyperbolic models.

Our paper studies a psychologically relevant limit case: we take the phrase 'instant gratification' literally, analyzing the case in which individuals prefer gratification in the present instant discretely more than consumption in the momentarily delayed future. This limit case is analytically tractable, and can easily be adapted for a range of applications. ${ }^{43}$ Finally, from the perspective of calibration, the instantaneous-gratification model serves as a good approximation for models in which the "present" lasts for as long as a month.

Christopher Harris, Faculty of Economics, University of Cambridge

David Laibson, Department of Economics, Harvard University, and National Bureau of Economic Research

\footnotetext{
${ }^{43}$ A partial list of applications that use our framework include Amador (2003), Della Vigna and Paserman (2005), Grenadier and Wang (2007), Bisin and Hyndman (2009), and Hsiaw (2010, 2010a), PalaciosHuerta and Pérez-Kakabadse (2011).
} 


\section{References}

Ainslie, George. 1992. Picoeconomics. Cambridge: Cambridge University Press.

Amador, Manuel. 2003. "A Political Economy Model of Sovereign Debt Repayment." Stanford University mimeo.

Angeletos, George-Marios, David Laibson, Andrea Repetto, Jeremy Tobacman, and Stephen Weinberg. 2001. "Hyperbolic discounting, wealth accumulation, and consumption." Forthcoming, Journal of Economic Perspectives.

Akerlof, George A. 1991. "Procrastination and Obedience." American Economic Review. (Papers and Proceedings), pp. 1-19.

Barro, Robert. 1999. "Laibson Meets Ramsey in the Neoclassical Growth Model." Quarterly Journal of Economics. 114(4), pp. 1125-52.

Bernheim, Douglas and Debraj Ray. 1987. "Economic Growth with Intergenerational Altruism," Review of Economic Studies 54 (2), April, pp. 227-242.

Bisin, Alberto and Kyle Hyndman. 2009. "Procrastination, Self-Imposed Deadlines and Other Commitment Devices," NYU mimeo.

Carroll, Christopher D. 1992. "The Buffer Stock Theory of Saving: Some Macroeconomic Evidence." Brookings Papers on Economic Activity. 2:1992, pp. 61-156.

Crandall, Michael, Hitoshi Ishii and Pierre-Louis Lions. 1992. "User's Guide to Viscosity Solutions", Bulletin of the American Mathematical Society, 27(1), pp. 1-67.

Deaton, Angus. 1991. "Saving and Liquidity Constraints." Econometrica. 59, pp. 122148.

Della Vigna, Stefano, and Ulrike Malmendier. 2004. "Contract Design and Self Control: Theory and Evidence," Quarterly Journal of Economics, May, 119, pp. 353-402.

Della Vigna, Stefano, and M. Daniele Paserman. 2005. "Job Search and Impatience," Journal of Labor Economics, July, Vol. 23, pp. 527-588.

Ekeland, Ivar and Ali Lazrak. 2006. "Being serious about non-commitment: subgame perfect equilibrium in continuous time," mimeo University of British Columbia. 
Ekeland, Ivar and Ali Lazrak. 2010. "The golden rule when preferences are time inconsistent," Mathematics and Financial Economics 4, pp. 29-55.

Grenadier, Steven R. and Neng Wang. 2007. "Investment under uncertainty and timeinconsistent preferences," Journal of Financial Economics, vol. 84(1), pages 2-39, April.

Harris, Christopher, and David Laibson. 2000. "Instantaneous Gratification," Harvard University mimeo.

Harris, Christopher, and David Laibson. 2001. "Dynamic Choices of Hyperbolic Consumers." Econometrica. 69(4), pp. 935-957.

Harris, Christopher and David Laibson. 2002. "Hyperbolic discounting and consumption," in eds. Mathias Dewatripont, Lars Peter Hansen and Stephen J. Turnovsky, Advances in Economics and Econometrics: Theory and Applications, Eighth World Congress, Volume I, pp. 258-98.

Hsiaw, Alice. 2010 "Goal-Setting, Social Comparison, and Self-Control," Harvard University mimeo. . 2010a. "Goal Bracketing and Self-Control," Harvard University mimeo.

Karatzas, Ioannis and Steven E. Shreve, Brownian Motion and Stochastic Calculus, Springer-Verlag, New York, 1988.

Karp, Larry. 2007. "Non-constant discounting in continuous time." Journal of Economic Theory 132, pp. 557-568.

Kimball, Miles. 1990. "Precautionary Saving in the Small and in Large," Econometrica, 58(1), pp. 53-73.

Krusell, Per and Tony Smith. 2000. "Consumption-Savings Decisions with QuasiGeometric Discounting," Econometrica.

Laibson, David. 1997. "Golden Eggs and Hyperbolic Discounting." Quarterly Journal of Economics. 62: 2, pp. 443-478.

, Andrea Repetto, and Jeremy Tobacman. 1998. "Self-Control and Saving for Retirement," Brookings Papers on Economic Activity 1, pp. 91-196. 
Laibson, David, Andrea Repetto, Jeremy Tobacman. 2011. "Estimating Discount Functions with Consumption Choices over the Lifecycle." Harvard mimeo.

Luttmer, Erzo and Thomas Mariotti. 2003. "Subjective Discounting in an Exchange Economy," Journal of Political Economy. 111, no. 5, pp. 959-89.

Maskin, Eric and Jean Tirole. 2001. "Markov Perfect Equilibrium," Journal of Economic Theory 100, 191-219.

McClure, Sam, Keith Ericson, David Laibson, George Loewenstein, and Jonathan Cohen. 2007. "Time Discounting for Primary Rewards." Journal of Neuroscience 27(21): $5796-5804$.

Merton, Robert C. 1971. "Optimum Consumption and Portfolio Rules in a ContinuousTime Model." Journal of Economic Theory 3 (December): 373-413.

Morris, Stephen. 2002. "Continuous Consumption Rules with Nonexponential Discounting," mimeo Yale University.

Morris, Stephen and Andrew Postlewaite. 1997. "Observational Implications of Nonexponential Discounting," mimeo.

O’Donoghue, Ted, and Matthew Rabin. 1999. "Doing It Now or Later." American Economic Review, 89(1), pp. 103-124.

Palacios-Huerta, Ignacio and Alonso Pérez-Kakabadse. 2011. "Consumption and Portfolio Rules with Stochastic Quasi-Hyperbolic Discounting" Brown University working paper series.

Phelps, E. S., and R. A. Pollak. 1968. "On Second-best National Saving and Gameequilibrium Growth." Review of Economic Studies. 35, pp. 185-199.

Rockafellar, R. Tyrrell. 1970. Convex Analysis. Princeton University Press: Princeton.

Strotz, Robert H. 1956. "Myopia and Inconsistency in Dynamic Utility Maximization." Review of Economic Studies. 23, pp. 165-180. 


\section{A. Proof of Lemma 2}

Note first that, if $x=0$, then one option for the current self is to consume at rate $y$ until the future arrives, at which point she will get the continuation payoff $\beta v_{\lambda}(0)$. This option yields the payoff

$$
\frac{u(y)+\lambda \beta v_{\lambda}(0)}{\gamma+\lambda}
$$

Second, as noted in the text, $w_{\lambda}-\beta v_{\lambda}$ is the value function of a consumer who: (i) has utility function $(1-\beta) u$; (ii) discounts the future exponentially at rate $\gamma+\lambda$; and (iii) has the exogenously given consumption function $c_{\lambda}$. Hence $w_{\lambda}-\beta v_{\lambda} \leq(1-\beta) \bar{e}_{\gamma+\lambda}$ for all $x \in[0, \infty)$, where $\bar{e}_{\gamma+\lambda}$ is the value function of a consumer who: (i) has utility function $u$; (ii) discounts the future exponentially at rate $\gamma+\lambda$; and (iii) chooses her consumption optimally. Hence

$$
\beta v_{\lambda} \geq w_{\lambda}-(1-\beta) \bar{e}_{\gamma+\lambda}
$$

for all $x \in[0, \infty)$. Third,

$$
\bar{e}_{\gamma+\lambda}(0)=\frac{1}{\gamma+\lambda} u(y)
$$

whenever $\lambda \geq \mu-\gamma$.

Combining these three observations, we obtain

$$
\begin{aligned}
w_{\lambda}(0) & \geq \frac{u(y)+\lambda \beta v_{\lambda}(0)}{\gamma+\lambda} \geq \frac{u(y)+\lambda\left(w_{\lambda}(0)-(1-\beta) \bar{e}_{\gamma+\lambda}(0)\right)}{\gamma+\lambda} \\
& =\frac{\lambda}{\gamma+\lambda} w_{\lambda}(0)+\frac{\gamma+\beta \lambda}{(\gamma+\lambda)^{2}} u(y)
\end{aligned}
$$

whenever $\lambda \geq \mu-\gamma$. Hence, rearranging,

$$
w_{\lambda}(0) \geq \frac{\gamma+\beta \lambda}{\gamma(\gamma+\lambda)} u(y)
$$

Finally, because there is no upper bound on consumption, we must have $w_{\lambda}^{\prime} \geq 0$. It follows that

$$
w_{\lambda} \geq w_{\lambda}(0)
$$

for all $x \in[0, \infty)$. Passing to the limit, we then obtain $\beta v=w \geq \frac{\beta}{\gamma} u(y)$ for all $x \in[0, \infty)$. The Lemma follows. 


\section{B. Proof of Theorem 4}

We begin with an overview of the proof. The first step is to use the optimality condition of the IG consumer to eliminate $c$ from her Bellman equation to yield what we call the reduced Bellman equation of the IG consumer (i.e. the Bellman equation of the IG consumer with $c$ substituted out). The second step is to use the optimality condition of the $\widehat{u}$ consumer to eliminate $\widehat{c}$ from her Bellman Equation to yield what we call the Reduced Bellman Equation of the $\widehat{u}$ consumer (i.e. the Bellman Equation of the $\widehat{u}$ consumer with $\widehat{c}$ substituted out). Third, we note that if we define $\widehat{u}_{+}$and $\widehat{u}_{0}$ as in Section 5.2 , then the Reduced Bellman Equation of the IG consumer is identical to the Reduced Bellman Equation of the $\widehat{u}$ consumer. Therefore the two reduced equations must have the same set of solutions, and the two consumers must have the same set of value functions.

Turning to the first step, for all $\alpha>0$ : let $f_{+}(\alpha)$ be the unique $c$ satisfying $u^{\prime}(c)=\alpha$; and put $h_{+}(\alpha)=u\left(f_{+}(\beta \alpha)\right)-\alpha f_{+}(\beta \alpha)$. Similarly, for all $\alpha \in \mathbb{R}$ : let $f_{0}(\alpha)$ be the unique $c$ satisfying $u^{\prime}(c)=\max \left\{u^{\prime}(y), \alpha\right\}$; and put $h_{0}(\alpha)=u\left(f_{0}(\beta \alpha)\right)-\alpha f_{0}(\beta \alpha)$. Finally, put

$$
h(\alpha, x)=\left\{\begin{array}{ll}
h_{+}(\alpha) & \text { if } x>0 \\
h_{0}(\alpha) & \text { if } x=0
\end{array}\right\}
$$

Then we may eliminate $c$ from the Bellman Equation of the IG consumer to obtain the differential equation

$$
0=\frac{1}{2} \sigma^{2} x^{2} v^{\prime \prime}+(\mu x+y) v^{\prime}-\gamma v+h\left(v^{\prime}, x\right)
$$

for $x \in[0, \infty)$ and the global bounds

$$
\frac{1}{\gamma} u(y) \leq v \leq \bar{v}
$$

We shall refer to equation (29) with global bounds (30) as the Reduced Bellman Equation of the IG consumer.

As for the second step, let $\widehat{u}_{+}, \widehat{u}_{0}$ and $\widehat{u}$ be given exactly as in Section 5.2. Then it can be checked that: (i) $\widehat{u}_{+}<u$ on $(0, \infty)$; and (ii) $\widehat{u}_{0} \leq u$ on $(0, y]$ with equality only at $y$. It follows that, like the value function of the IG consumer, the value function $\widehat{v}$ of the $\widehat{u}$ consumer satisfies $\widehat{v} \leq \bar{v}$. It can also be checked that $\lim _{\widehat{c} \rightarrow \infty} \widehat{u}_{+}(\widehat{c})=\lim _{c \rightarrow \infty} u(c)$. Hence there exists $b \in(y, \infty)$ such that $\widehat{u}_{+}(b)=u(y)$. Hence, if the $\widehat{u}$ consumer consumes $b$ when $x>0$ and $y$ when $x=0$, then she will obtain a payoff of $\frac{1}{\gamma} u(y)$. It follows that 
$\widehat{v} \geq \frac{1}{\gamma} u(y)$. Overall, then, the Bellman Equation of the $\widehat{u}$ consumer takes the form of the differential equation

$$
0=\frac{1}{2} \sigma^{2} x^{2} \widehat{v}^{\prime \prime}+(\mu x+y-\widehat{c}) \widehat{v}^{\prime}-\gamma \widehat{v}+\widehat{u}(\widehat{c}, x)
$$

for $x \in[0, \infty)$, the optimality condition

$$
\left\{\begin{array}{ll}
\frac{\partial \widehat{u}}{\partial \widehat{c}}(\widehat{c}, x)=\widehat{v}^{\prime} & \text { if } x>0 \\
\frac{\partial \widehat{u}}{\partial \widehat{c}}(\widehat{c}, x)=\max \left\{\widehat{u}_{0}^{\prime}(y), \widehat{v}^{\prime}\right\} & \text { if } x=0
\end{array}\right\}
$$

and the global bounds

$$
\frac{1}{\gamma} u(y) \leq \widehat{v} \leq \bar{v} .
$$

For all $\alpha>0$ : let $\widehat{f}_{+}(\alpha)$ be the unique $\widehat{c}$ satisfying $\widehat{u}_{+}^{\prime}(\widehat{c})=\alpha$; and put $\widehat{h}_{+}(\alpha)=$ $\widehat{u}_{+}\left(\widehat{f}_{+}(\alpha)\right)-\alpha \widehat{f}_{+}(\alpha)$. Similarly, for all $\alpha \in \mathbb{R}$ : let $\widehat{f}_{0}(\alpha)$ be any $\widehat{c}$ satisfying $\widehat{u}_{0}^{\prime}(\widehat{c})=$ $\max \left\{\widehat{u}_{0}^{\prime}(y), \alpha\right\} ; 4$ and put $\widehat{h}_{0}(\alpha)=\widehat{u}_{0}\left(\widehat{f}_{0}(\alpha)\right)-\alpha \widehat{f}_{0}(\alpha)$ (which is uniquely defined even when $\widehat{f}_{0}(\alpha)$ is not). Finally, put

$$
\widehat{h}(\alpha, x)=\left\{\begin{array}{ll}
\widehat{h}_{+}(\alpha) & \text { if } x>0 \\
\widehat{h}_{0}(\alpha) & \text { if } x=0
\end{array}\right\} .
$$

Then we may eliminate $\widehat{c}$ from the Bellman Equation of the $\widehat{u}$ consumer, namely (31-33), to obtain the differential equation

$$
0=\frac{1}{2} \sigma^{2} x^{2} \widehat{v}^{\prime \prime}+(\mu x+y) \widehat{v}^{\prime}-\gamma \widehat{v}+\widehat{h}\left(\widehat{v}^{\prime}, x\right)
$$

for $x \in[0, \infty)$ and the global bounds

$$
\frac{1}{\gamma} u(y) \leq v \leq \bar{v} .
$$

We shall refer to equation (34) with global bounds (35) as the Reduced Bellman Equation of the $\widehat{u}$ consumer.

Finally, it is easy to see that equations (29) and (34) will be identical iff the functions $h$ and $\widehat{h}$ are the same. Moreover, as can be shown by direct calculation, this is indeed the

\footnotetext{
${ }^{44}$ In terms of $\widehat{u}_{+}$and $\widehat{f}_{+}$, we have: $\widehat{f}_{0}(\alpha)=\widehat{f}_{+}(\alpha)$ if $\alpha>\widehat{u}_{+}^{\prime}(\psi y) ; \widehat{f}_{0}(\alpha) \in[\psi y, y]$ if $\alpha=\widehat{u}_{+}^{\prime}(\psi y)$ and $\widehat{f}_{0}(\alpha)=y$ if $\alpha<\widehat{u}_{+}^{\prime}(\psi y)$.
} 
case for the given choice of $\widehat{u}_{+}, \widehat{u}_{0}$ and $\widehat{u}$. Hence the Reduced Bellman Equation of the IG consumer is identical to the Reduced Bellman Equation of the $\widehat{u}$ consumer, as required.

\section{Proof of Theorem 5}

The proof of Theorem 4 in Appendix B above shows that $v$ satisfies the Reduced Bellman Equation of the IG consumer (i.e. the Bellman Equation of the IG consumer with $c$ substituted out) iff $v$ satisfies the Reduced Bellman Equation of the $\widehat{u}$ consumer (i.e. the Bellman Equation of the $\widehat{u}$ consumer with $\widehat{c}$ substituted out). Furthermore, standard considerations show that $v$ satisfies the Reduced Bellman Equation of the $\widehat{u}$ consumer iff $v$ is the value function of the optimization problem of the $\widehat{u}$ consumer. More explicitly, $v$ satisfies the Reduced Bellman Equation of the $\widehat{u}$ consumer iff, for all $x \in[0, \infty), v(x)$ is the supremum of all payoffs that are feasible for the $\widehat{u}$ consumer when her initial wealth is $x$. This already yields both existence and uniqueness of $v$, for the simple reason that the supremum of any set of numbers exists and is unique. In particular, the supremum of all the feasible payoffs of the $\widehat{u}$ consumer exists and is unique. Turning to the consumption function, we recall that $u^{\prime}(c)=\beta v^{\prime}$ if $x>0$ and $u^{\prime}(c)=\max \left\{u^{\prime}(y), \beta v^{\prime}\right\}$ if $x=0$. Since $u$ is strictly concave (and therefore $u^{\prime}$ is invertible), the existence and uniqueness of $c$ follows directly from the existence and uniqueness of $v$.

\section{Proof of Theorem 8}

The basic idea behind the proof is to view $v_{\sigma}$ as the value function of the optimization problem of the stochastic $\widehat{u}$ consumer and $v$ as the value function of the optimization problem of the deterministic $\widehat{u}$ consumer. There are several ways of implementing this idea. One way is to note that the dynamics of the problem depend continuously on $\sigma$, and that the utility function $\widehat{u}$ is upper semicontinuous. (It is continuous except at $x=0$, where it may jump up in the limit as $x \downarrow 0$, because $\widehat{u}_{0} \geq \widehat{u}_{+}$.) From this it follows at once that $\lim \sup _{\sigma \downarrow 0} v_{\sigma}(x) \leq v(x)$ for all $x \in[0, \infty)$. On the other hand, explicit consideration of the form of the optimal consumption function $\widehat{c}$ of the deterministic $\widehat{u}$ consumer shows that $\lim _{\sigma \downarrow 0} v_{\sigma}(x ; \widehat{c})=v(x)$ for all $x \in[0, \infty)$, where $v_{\sigma}(x ; \widehat{c})$ denotes the payoff to the stochastic $\widehat{u}$ consumer when she employs the consumption function $\widehat{c}$. Specifically: if $\mu<\frac{1}{\beta} \gamma$, then there exists $\varepsilon>0$ such that $\widehat{c}$ is continuous on $(0, \varepsilon)$ and $\widehat{c}(0+)=\psi \bar{c}>y$, where $\psi=\frac{\rho-(1-\beta)}{\rho}$; and, if $\mu \geq \frac{1}{\beta} \gamma$, then $\widehat{c}$ is continuous on $[0, \infty)$ and $\widehat{c}(0) \leq \psi y<y$. In particular, $\liminf \operatorname{in\downarrow }_{\sigma v_{\sigma}}(x) \geq v(x)$. 


\section{Figure 1: Sequential generations of autonomous selves.}

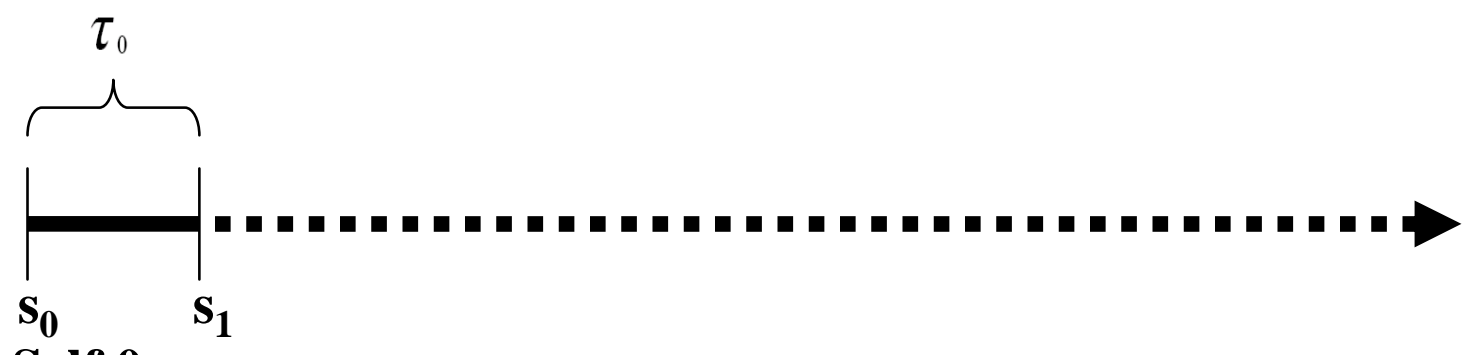

Self 0:

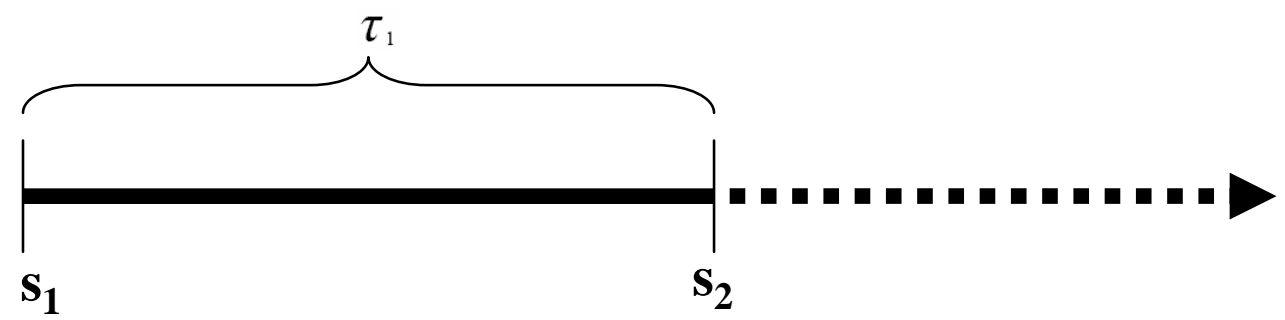

Self 1:

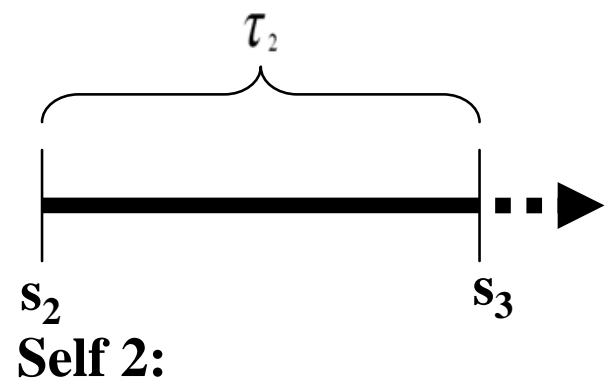

The span of control (solid line) of self $n$ lasts from its time of birth $\left(t=s_{n}\right)$ to the time of birth of self $n+1\left(t=s_{n+1}\right)$. The length of this control period, $s_{n+1}-s_{n}$, is the stochastic variable $\tau_{\mathrm{n}}$, which has an exponential distribution. 


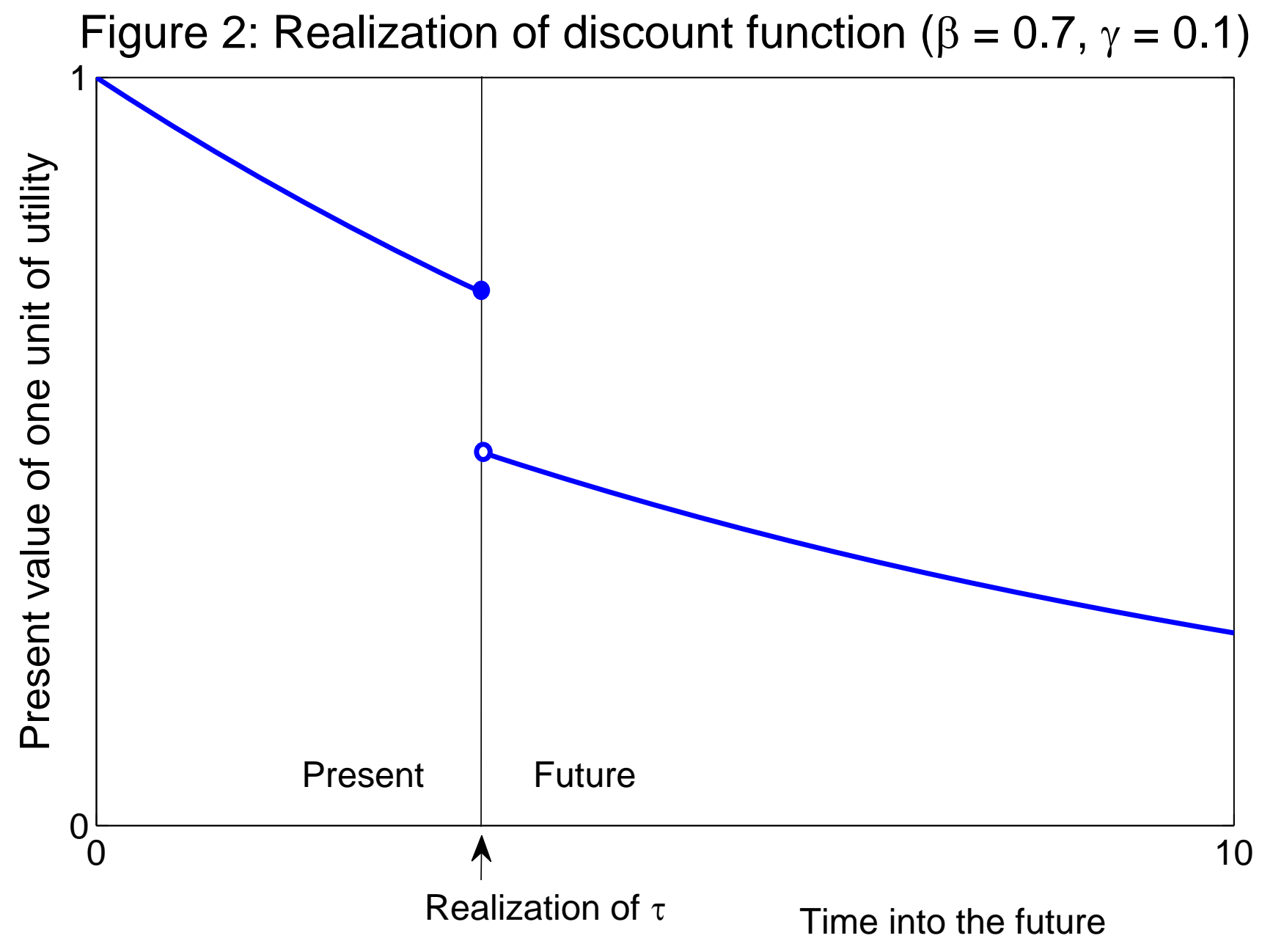

The discount function represents the present value of one unit of future utility. The discount function discretely drops when the present ends and the future begins. This present-to-future transition occurs at a stochastic time. Figure 2 shows a particular realization of this transition. 


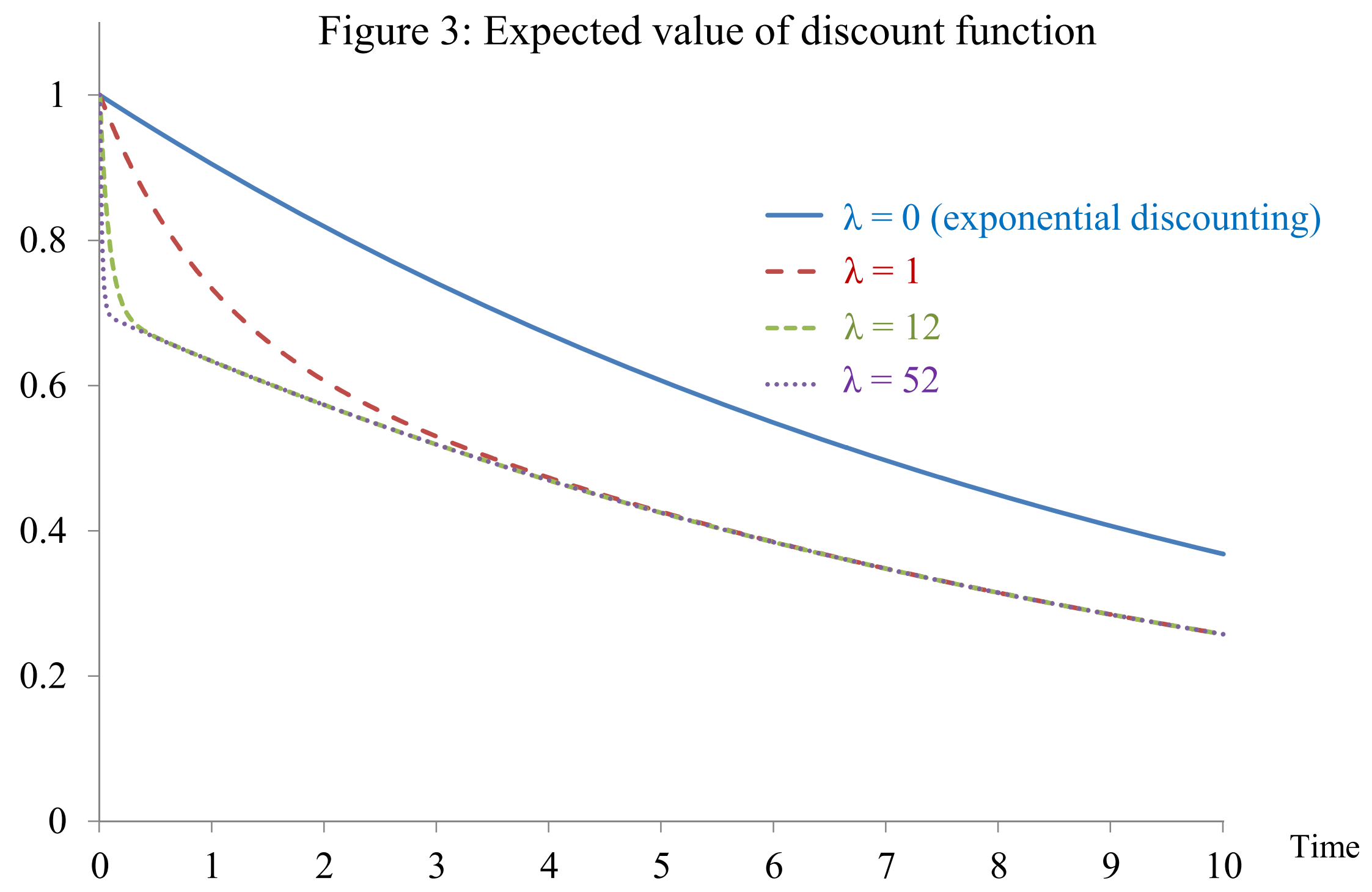

The expected value of the discount function is plotted for $\beta=0.7$ and $\gamma=0.1$. With $\lambda=0$, the future never arrives and the function is $\exp (-\gamma t)$. As long as $\lambda$ is strictly positive, the future will eventually arrive so the function asymptotes to $\beta \exp (-\gamma t)$. The higher the value of $\lambda$ the faster the function asymptotes. 
Figure 4: graph of $u, \hat{u}_{+}$and $\hat{u}_{0}$

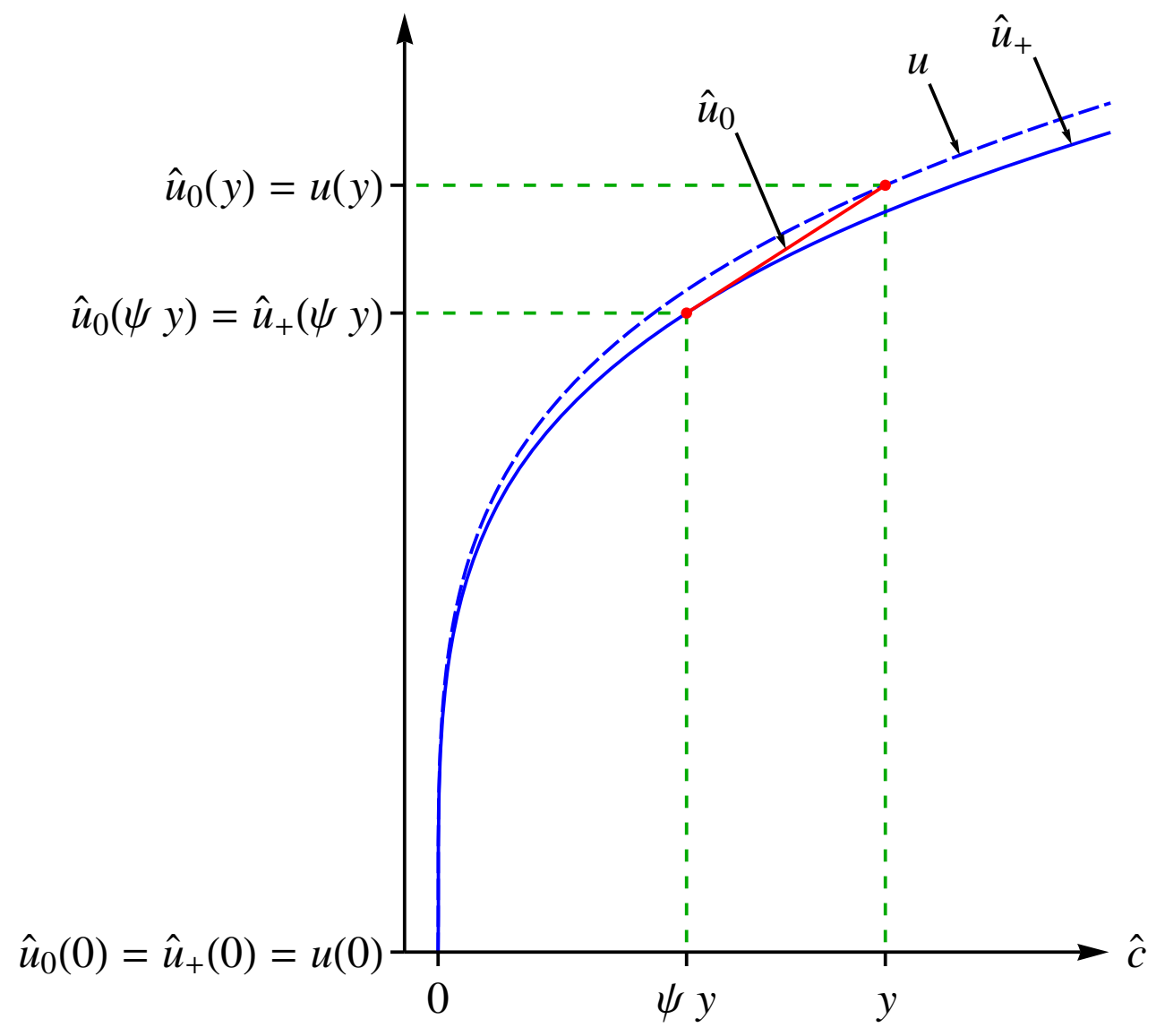

The relationship between $u, \hat{u}_{+}$and $\hat{u}_{0}$ is plotted for the case $\beta=\frac{2}{3}, \rho=\frac{3}{4}$ and $y=1$. Utility function $u$ has constant relative risk aversion $\rho$. Utility function $\hat{u}_{+}$is given by $\frac{\psi}{\beta} u\left(\frac{\hat{c}}{\psi}\right)+\frac{\psi-1}{\beta}$, where $\psi=\frac{\rho-(1-\beta)}{\rho}$. Utility function $\hat{u}_{0}$ coincides with $\hat{u}_{+}$up to $\psi y$ and thereafter with the tangent to $\hat{u}_{+}$at $\psi y$. 
Figure 5: Consumption functions for $\mu \in\{0.04,0.07,0.09\}$

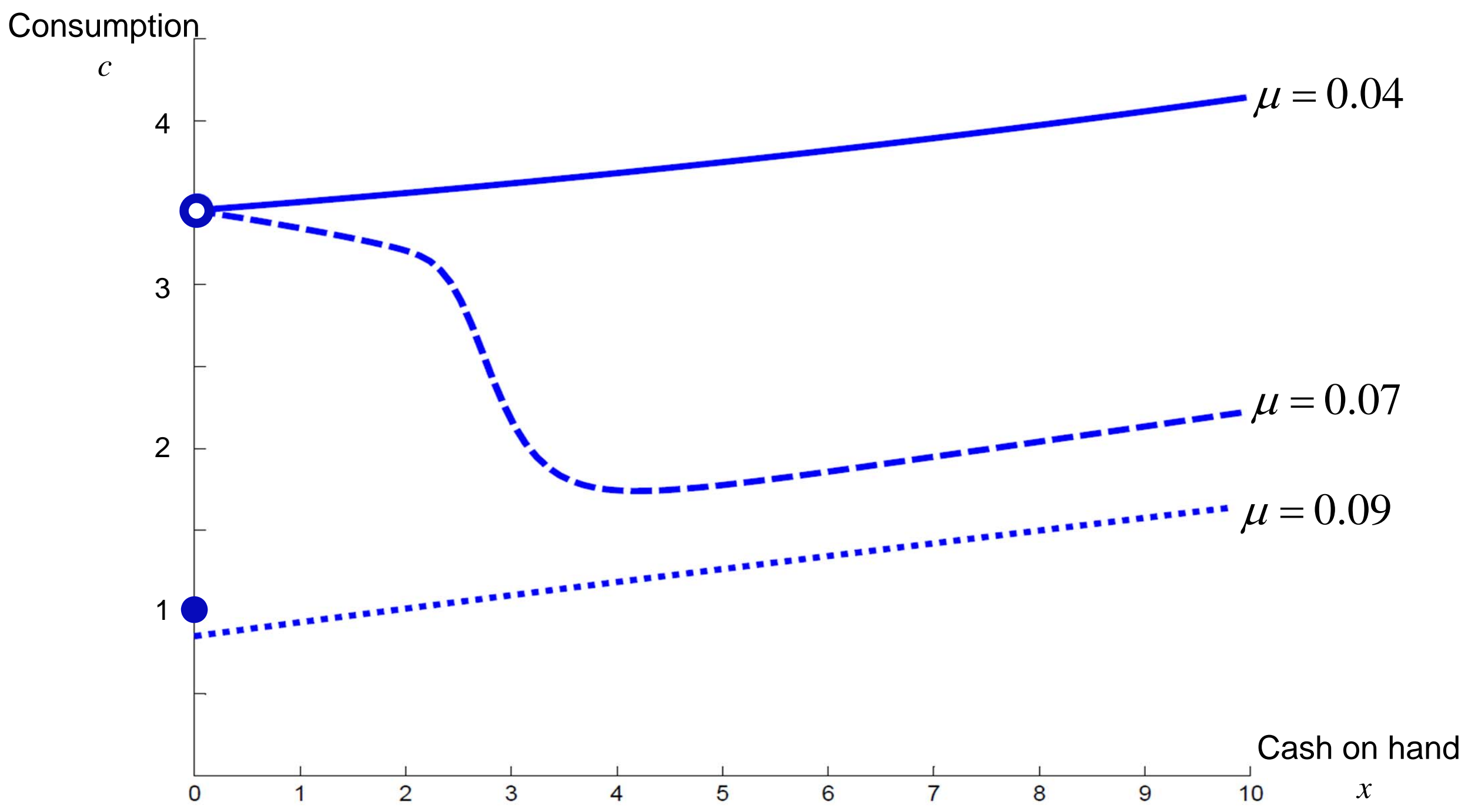

These consumption functions were generated in Matlab by numerically solving the Bellman System of the IG consumer (with $\beta=2 / 3, \gamma=0.05, \sigma=0.17, \rho=3 / 4, y=1$ ). The top two cases have a discontinuity at $x=0$. 